Portland State University

PDXScholar

Civil and Environmental Engineering Master's

Project Reports

Summer 2020

\title{
Ultrasonic Coda Wave Comparison for Quality Control of Manufactured Parts: Proof of Feasibility
}

Alexei Marin-Cortes

Portland State University

Follow this and additional works at: https://pdxscholar.library.pdx.edu/cengin_gradprojects

Part of the Civil Engineering Commons

Let us know how access to this document benefits you.

\section{Recommended Citation}

Marin-Cortes, Alexei, "Ultrasonic Coda Wave Comparison for Quality Control of Manufactured Parts: Proof of Feasibility" (2020). Civil and Environmental Engineering Master's Project Reports. 51.

https://doi.org/10.15760/CCEMP.50

This Project is brought to you for free and open access. It has been accepted for inclusion in Civil and Environmental Engineering Master's Project Reports by an authorized administrator of PDXScholar. Please contact us if we can make this document more accessible: pdxscholar@pdx.edu. 


\title{
ULTRASONIC CODA WAVE COMPARISON FOR QUALITY CONTROL OF MANUFACTURED PARTS: PROOF OF FEASIBILITY
}

\author{
Alexei Marin, M.S. Student \\ Thomas Schumacher, $\mathrm{PhD}$, Associate Professor (Adviser) \\ Portland State University, \\ Portland, OR \\ Abstract: Manufactured parts are a staple of modern urban life, visible in homes (e.g., kitchen \\ appliances), offices (e.g., printers), the automobile and aerospace industries (e.g., body parts), \\ and more. This M.S. project intended to determine whether ultrasonic testing could be used in a \\ simple and rapid manner to perform quality control of manufactured parts. Ultrasonic testing is \\ employed for this purpose using two surface-coupled transducers, one being a transmitter and the \\ other one a receiver. An ultrasonic pulse, which is generated by the transmitting transducer, \\ propagates through the part, collecting information about material and geometry, as well as \\ potential unwanted features. The resulting response is recorded at the receiving transducer for a \\ long duration, i.e. until the wave amplitude has dampened out. Coda wave analysis, which \\ studies the later portions of a recorded signal, is then used to compute a similarity index between \\ a signal from a part and a signal from a reference part that has been examined and approved. \\ Coda wave fields have been found significantly more sensitive to minute differences in two \\ signals compared to the first wave arrival. This MS project report introduces the setup and \\ instrumentation, testing methodology, coda wave analysis and similarity indices, and discusses \\ the feasibility of the proposed approach on a set of manufactured parts made of different \\ materials with different alterations.
}




\section{ULTRASONIC CODA WAVE COMPARISON FOR QUALITY CONTROL OF MANUFACTURED PARTS: PROOF OF FEASIBILITY}

\section{Introduction/Background}

Manufactured parts are ubiquitous in modern urban life, visible in homes (e.g., kitchen appliances), offices (e.g., pens, printers), the automobile and aerospace industries, and more. Manufactured parts are defined as physical elements produced in a mechanized manufacturing process, where the elements are either a part of larger product or the final product by itself. These parts can be manufactured of a myriad of materials. They can be of a single material, such as a wood block, steel plate, or a single polymer piece, or they can be a composite, consisting of multiple integrated materials such as a CFRP laminate. Numerous techniques have been developed for the quality control of these parts. The techniques include physical and chemical principles, ranging from laser-induced spectroscopy to measurement of oxygen to electromagnetic spectroscopy [1]. The techniques apply for traditional subtractive manufacturing as well as the emerging additive manufacturing process [2].

One common non-destructive testing (NDT) technique is ultrasound testing, which uses the principle of ultrasonic stress wave propagation in a material. Ultrasound testing to study the health of materials has been widely used and developed. It is a technique existing since middle of the $20^{\text {th }}$ century [3]. One instance of ultrasound use is in testing to detect flaws in concrete of civil structures, using a practice called ultrasonic echo testing [4]. Similarly, ultrasounds are currently able to study the condition of manufactured parts, such as using laser ultrasound waves in metal additive manufacturing [5].

Ultrasound stress waves are able to capture many characteristics as they propagate and interact with a material. Some studies focus on the time-of-flight (TOF) of an ultrasonic pulse, while others focus on the vibration periods presented in the received waveform [6]. Other various studies create a map of the structure using an array of ultrasonic transducers, usually of 30 or more to be accurate [7]. While such methods aim to create a detailed image of the element, they are more time and cost consuming.

For this study, the focus is to study the coda wave (also known as diffuse field) of the received ultrasonic stress wave as it traverses a material. The setup employs a single emitter and a single receiver. The coda wave is looked on as the later, lower amplitude portion of a received signal [8]. In this case, an ultrasonic wave that traverses a material, will contain specific and unique information about the material in the tail end of the received signal. Coda waves have been studied for various materials, such as steel [9], fiber reinforced composites [10], and concrete [11].

Monitoring of coda waves to examine alterations in structures over time has been explored for various materials. Some experimentation researches the variation of coda waves in carbon-fiber reinforced polymer (CFRP) specimens as they develop fractures [3]. The experiments present an instrumentation where sensors are semi-permanent or permanently adhered to the specimen. While minimizing measurement variability and uncertainty, this approach requires that each specimen has its own set of sensors. In contrast, this study assumes that a set of the same 
transducers are coupled to each part in a quick and consistent manner. Ideally, the transducer coupling would be performed by a robotic system.

This robotic procedure will still result in variability in the measurement process due to slight variations in coupling conditions. The coupling conditions affect the acoustic energy and acoustic transmissivity in the material, thus affecting the received signal [12]. This is a particular challenge for the coda wave portion, which is sensitive to minute changes in the setup. A study of the effect of coupling force is part of this research study.

Other studies employ non-contact, i.e. air-coupled ultrasound, incorporating the travel of the wave through air [13]. This method works best with the surface (or Rayleigh) waves traveling through the material. Rayleigh waves propagate near the surface and thus provide limited information with respect to depth. Also, the ultrasonic transducers used for these experiments require a higher resolution instrumentation for capturing useful signals [14]. This study intends to make use of inexpensive equipment to increase both the efficiency as well as the marketability and appeal of testing method to the manufacturing industry.

Overall, the manufacturing industry is searching to adapt existing the NDT techniques, including ultrasound testing, to the current manufacturing environment. One such effort is into in-line process testing and monitoring, where the testing technique evaluates the health of the part as it is being processed. Testing a part during production allows to detect and correct parts with anomalies at an earlier stage, thus reducing cost and time of production. However, the techniques in use today present limitations for many kinds of parts. In particular, difficulties with manufactured part geometry and microstructure, along with standardization in the manufacturing process exist with the existing techniques [15]. These difficulties apply to traditional manufacturing, e.g, subtractive manufacturing, and the contemporary processes, e.g., additive manufacturing. There is a pursuit and demand for novel, efficient and inexpensive NDT techniques to aid the manufacturing quality control process. 


\section{Research Significance and Objectives}

The objective of this study was to investigate the feasibility of an effective ultrasonicbased tool for quality control of mass manufactured parts. The setup consists of one transmitting transducer, which emits an ultrasonic pulse, and a receiving transducer, which records the ultrasonic stress wave after it has propagated through a part. The main interest is on the coda wave portion of the recorded signals, which has been shown to be extremely sensitive to minute changes in a material. Parameters affecting the measurements such as coupling force of the transducers as well as different types of alterations are introduced in a variety of ways and on select manufactured parts made of different materials to evaluate the feasibility of the proposed approach for quality control purposes. The proposed approach has the potential to be inexpensive, fast, reliable, and computationally efficient in detecting small differences between a tested part and an approved reference part.

These characteristics of the proposed approach make it appealing to the current manufacturing environment. Its efficiency allows it to integrate ideally the in-line portion of the process, providing a quality control assessment from an early stage. Also, the small size and portability of the transducers have the potential to adapt to multiple kinds of part geometries. This would allow it to evaluate complex shapes, which are difficult to examine under existing NDT processes. 


\section{Theoretical Background}

\section{Ultrasonic Coda Waves}

The term coda wave has existed and evolved since the mid-60s in the study of earthquake waves, referring to the latter arriving portion of a seismic wave [8]. Coda waves have been used further to study stresses in different materials over the last ten years. For instance, coda wave interferometry, which measures changes in wave velocity and phase shifts across the coda portion, has been used to describe stress variations in concrete [11].

Fundamentally, the coda wave portion consists of multiple superimposed waves caused by reflection and scattering in a material [8]. This is opposed to the first, i.e. coherent portion of a stress wave. The coda segment of a recorded signal displays a lower amplitude due to significant attenuation of the initial wave. It is often referred to as the diffuse field, a term mentioned in the field of acoustic-ultrasonic as an ultrasonic wave traveling across a scattering medium [18]. Both terms share the commonality of wave scattering and propagation over alternative and multiple paths before arriving at a receiving transducer.

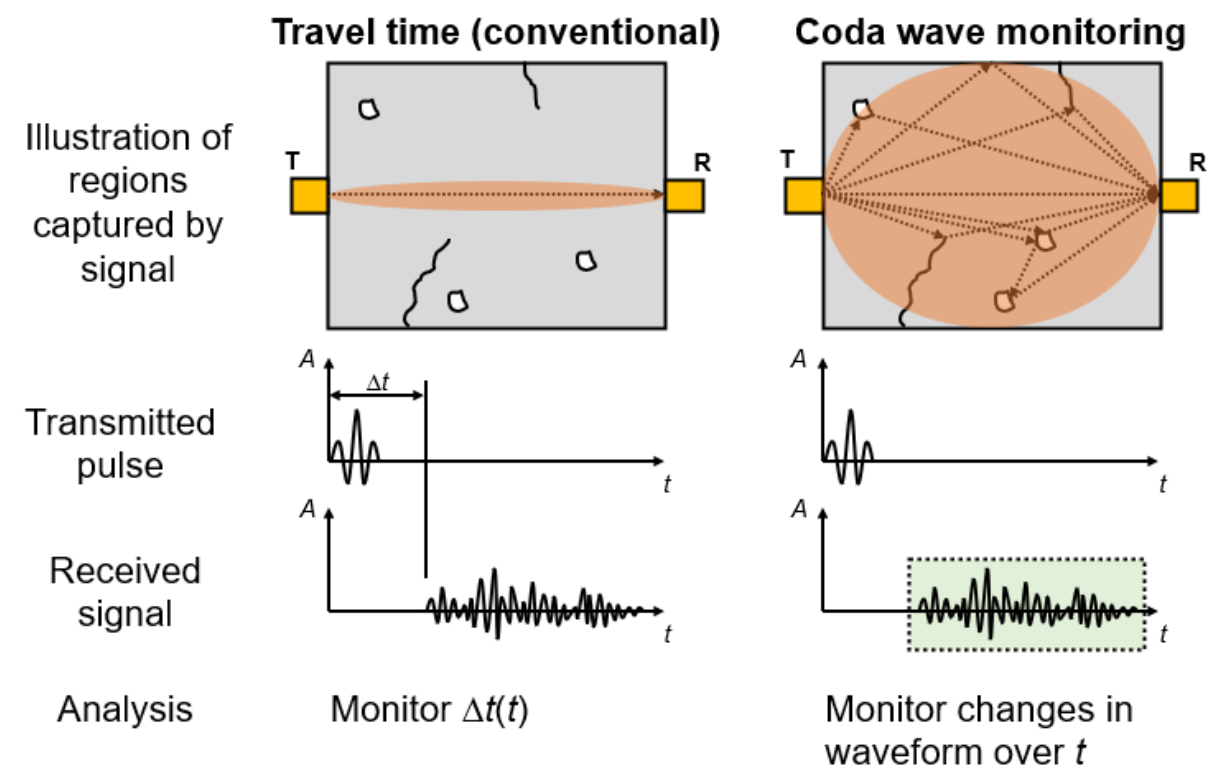

Figure III-1. Conventional vs coda wave monitoring (Courtesy of Thomas Schumacher)

Figure III-1 illustrates the difference between traditional ultrasonic testing (left column) and coda wave monitoring (right column). In traditional ultrasonic testing, the changes in the structure are evaluated by observing the time delay between the moment the pulse is excited from the transmitter to the moment the signal arrives at the receiver. The signal travels a single, most direct path between transmitter and receiver during this time, thus this path is the only region in the structure the testing is examining. In coda wave monitoring, the changes are monitored over the length of the waveform. The length captures the scattering of the pulse against the various 
boundaries of the material, hence portraying a much larger area of the specimen.
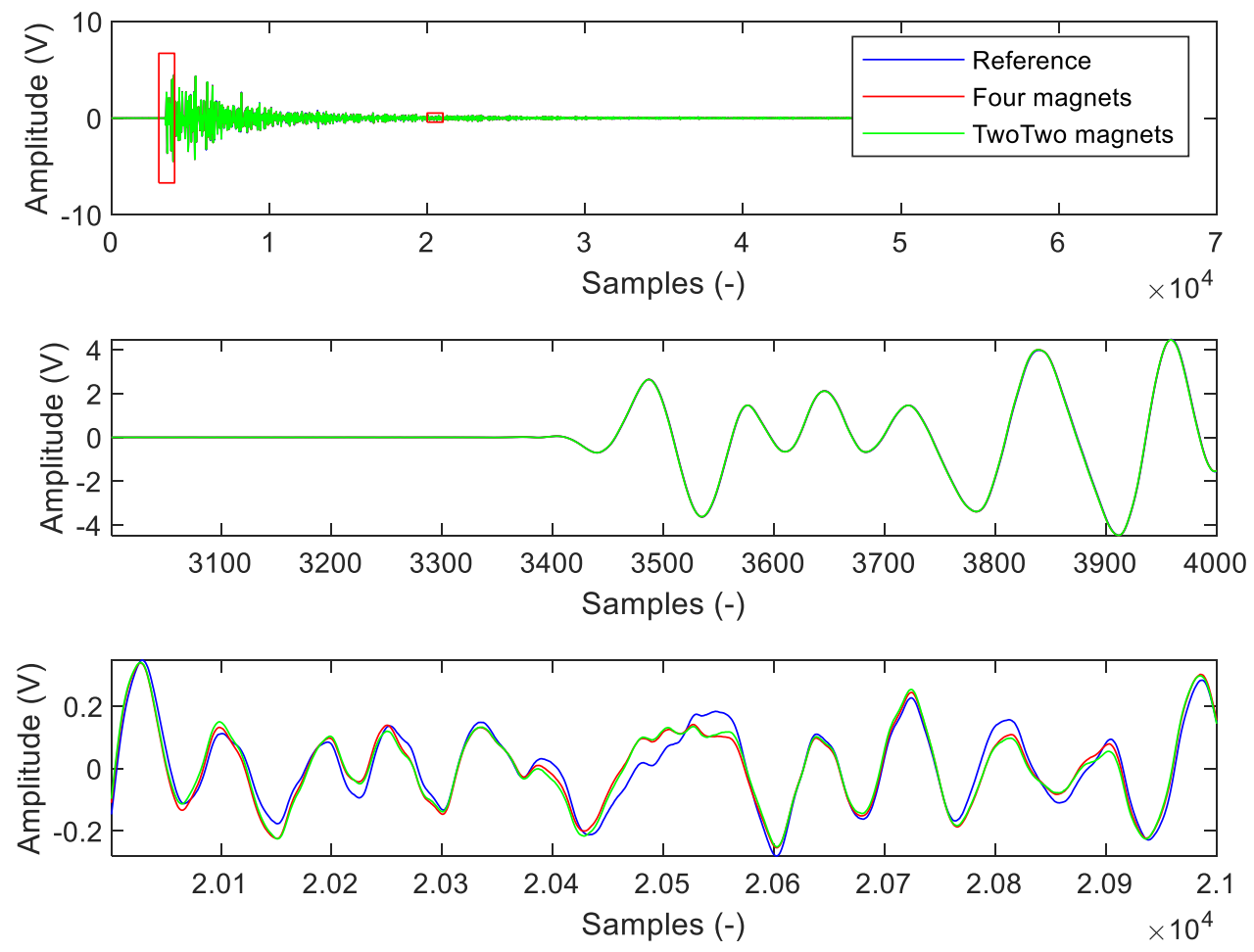

Figure III-2. Sample ultrasonic stress wave recorded in a manufactured part for three different test conditions: over its entirety (a), arrival (i.e., coherent) portion (b), and coda wave portion (c).

Figure III-2. Sample ultrasonic stress wave recorded in a manufactured part for three different test conditions: over its entirety (a), arrival (coherent) portion (b), and coda wave portion (c)Figure III-2 shows sample responses of an identical pulse through the same part for the reference case and two different altered conditions. The transmitted pulse is a $50 \mathrm{kHz}$ sine-type pulse. It can be observed that the coherent portion shown in Figure III-2(b) is virtually identical to the naked eye for all three measurements. Conversely, the latter portion, i.e. the coda wave, which is shown in Figure III-2(c) shows differences between the three measurements. These sample recordings are part of "Test 2: Steel Coupler Nut" and are discussed in detail in the corresponding sections.

\section{Signal Processing Methods}

Digital signal processing (DSP) methods are used in this study to load, filter, process, and analyze signals. One initial step used was detrending, where an average value over a portion of the signal prior to the wave arrival is subtracted from the signal. A procedure used in some cases was stacking, where multiple measurements taken under the same conditions are averaged. This reduces the noise and hence increases the signal-to-noise ratio (SNR). Finally zero-padding was employed, i.e. data points with a value of " 0 " are added at the end of a signal. The additional points increase the number of samples, which allows a Fourier analysis to fit additional coefficients, essentially increasing the resolution in the frequency spectrum. 
The signal recordings are analogous to arrays of voltage amplitudes. The diversity of studies in the field use a wide range of methods to compare, based on diverse fields such as statistics and algebra [19]. This study relies on statistical parameters to compare two arrays with each other. Such statistics generate a single number to describe the affinity between two signals. Two parameters, which can be thought of as similarity indices, were employed.

The coefficient of determination $\left(R^{2}\right)$ was the first candidate similarity index considered. It is a magnified number of the correlation coefficient, which is a measure of the strength of the relationship between two variables. By squaring the correlation coefficient into the coefficient of determination, it ensures the measure is consistently positive. $R^{2}$ is defined as the covariance of an "altered" signal $Y_{i}$ and "reference" $Y_{0}$ divided by the product of their independent standard deviations [10]. $R^{2}$ ranges between 0 and 1 , representing none and complete correlation, respectively:

$$
\begin{aligned}
& \rho_{Y_{0}, Y_{i}}=\rho=\frac{\operatorname{CoV}\left(Y_{0}, Y_{i}\right)}{\sigma_{Y_{0}} \sigma_{Y_{i}}}=\frac{\sigma_{Y_{0}, Y_{i}}}{\sigma_{Y_{0}} \sigma_{Y_{i}}} \\
& R^{2}=\rho^{2}=M(\lambda, A) \Delta \sigma
\end{aligned}
$$

\section{Figure III-3. Correlation coefficient (a) and coefficient of determination (b) equations.}

The Magnitude-Squared Coherence (MSC) estimate is another candidate index for comparing signals. MSC evaluates the similarity between two arrays of values in their frequency domains. It converts the recordings linear in the time spectrum and translates them into their frequency content. The measure is a function of the cross-power spectral density of the two arrays. The density provides a statistical description of the signals in the frequency spectrum [20]. It proceeds to compare the two signal contents over a range of frequencies up to the Nyquist rate. The Nyquist rate is defined as one half of the sampling rate of the recording. MSC is defined as follows:

$$
\operatorname{MSC}=\left|C_{Y o Y i}\left(e^{j \omega}\right)\right|^{2}=\frac{\left|R_{Y 0 Y i}\left(e^{j \omega}\right)\right|^{2}}{R_{Y 0}\left(e^{j \omega}\right) R_{Y i}\left(e^{j \omega}\right)}
$$

\section{Figure III-4. Magnitude squared coherence equation}

In order to produce one number using MSC, values of the MSC function can either be integrated between two select frequency values (lower and upper bound), or an MSC value at a specific frequency can be picked. MSC-based similarity indices have one major advantage over the $R^{2}$ index as they are not sensitive to small time shifts between measurements. 


\section{Experimental Setup}

In this section, the test setup and specimens are described in detail. The criteria for selecting test specimens is centered on their commercial availability and representativeness of typical mass-manufactured parts.

\section{Test setup}

The experimental setup for the project is shown in Figure IV-1 and serves to perform the ultrasonic stress wave measurements for a variety of different test specimens. An arbitrary waveform generator (BK Precision 4053) creates a one-cycle sine pulse, having a frequency of 50 or $100 \mathrm{kHz}$ (depending on the test specimen) at $20 \mathrm{Vpp}$ (Volt, peak-to-peak), as shown in Figure IV-2, and transmits it into the specimen through the transmitting transducer (T). The receiving transducer $(\mathrm{R})$ captures the response, such as the response in Figure IV-3, which is then intensified by a pre-amplifier (Olympus, Model 5660B) to a high-speed data recorder (Elsys, Model TraNET FE). This recorder triggers on the transmitted pulse, digitizes and transmits the signal to a PC laptop for storage and data analysis. The number of samples recorded per signal ranges between 32,768 and 131,072, depending on the test specimen. The sampling rate for all tests was $10 \mathrm{MHz}$ with a low-pass filter set to $1 \mathrm{MHz}$ to avoid aliasing.

The transducers are equivalent to microphones capable of generating and capturing ultrasonic waves traveling through a test specimen. The transducers are Panametrics, Model V103 normalwave transducers, which use the principle of piezoelectricity. As such, they can both actuate (i.e., vibrate when exposed to a voltage) and receive (i.e., produce a voltage output when strained). The coupling of the transducers was done by means of mounting clamps without the use of a couplant, i.e. dry-coupling.

Additional details for each test, along with a description of the test specimens, are provided in the subsequent sections.

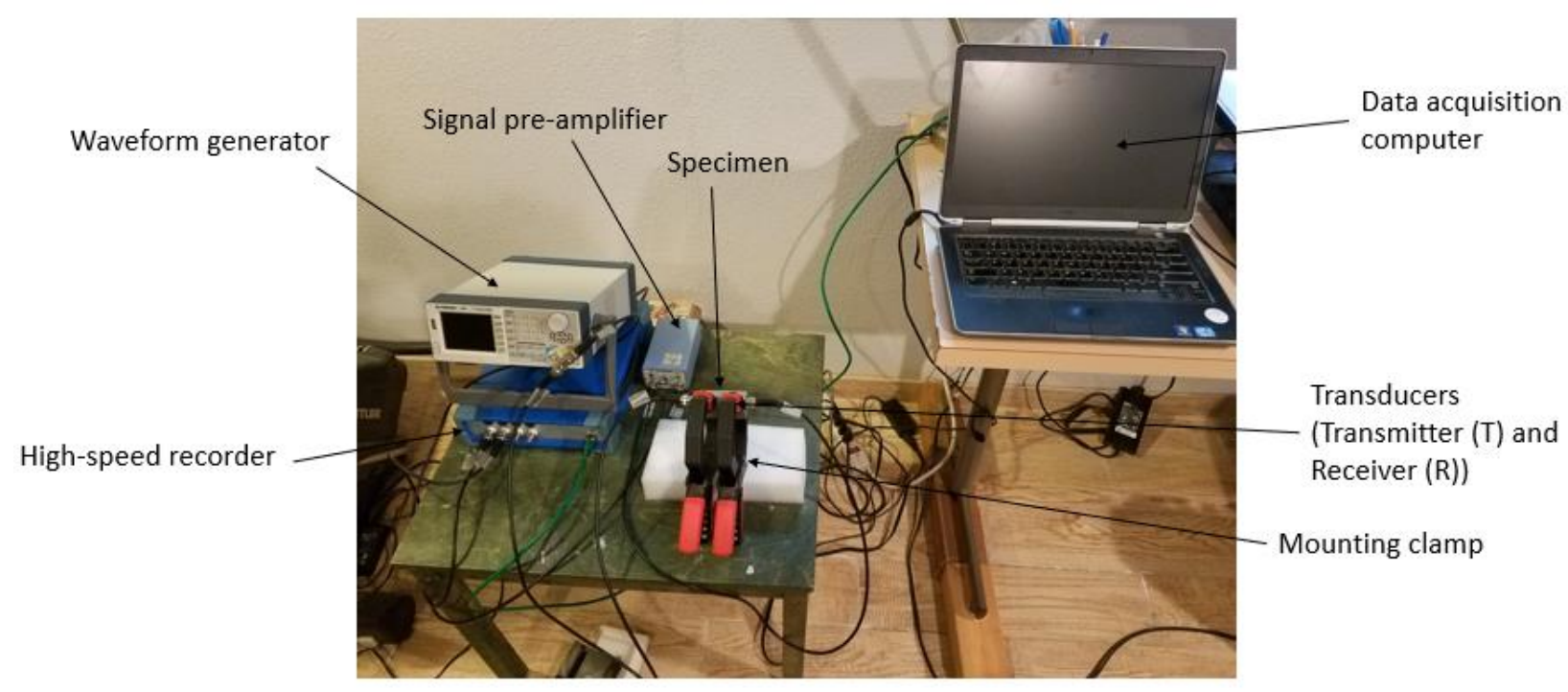

Figure IV-1. Image of experimental setup 


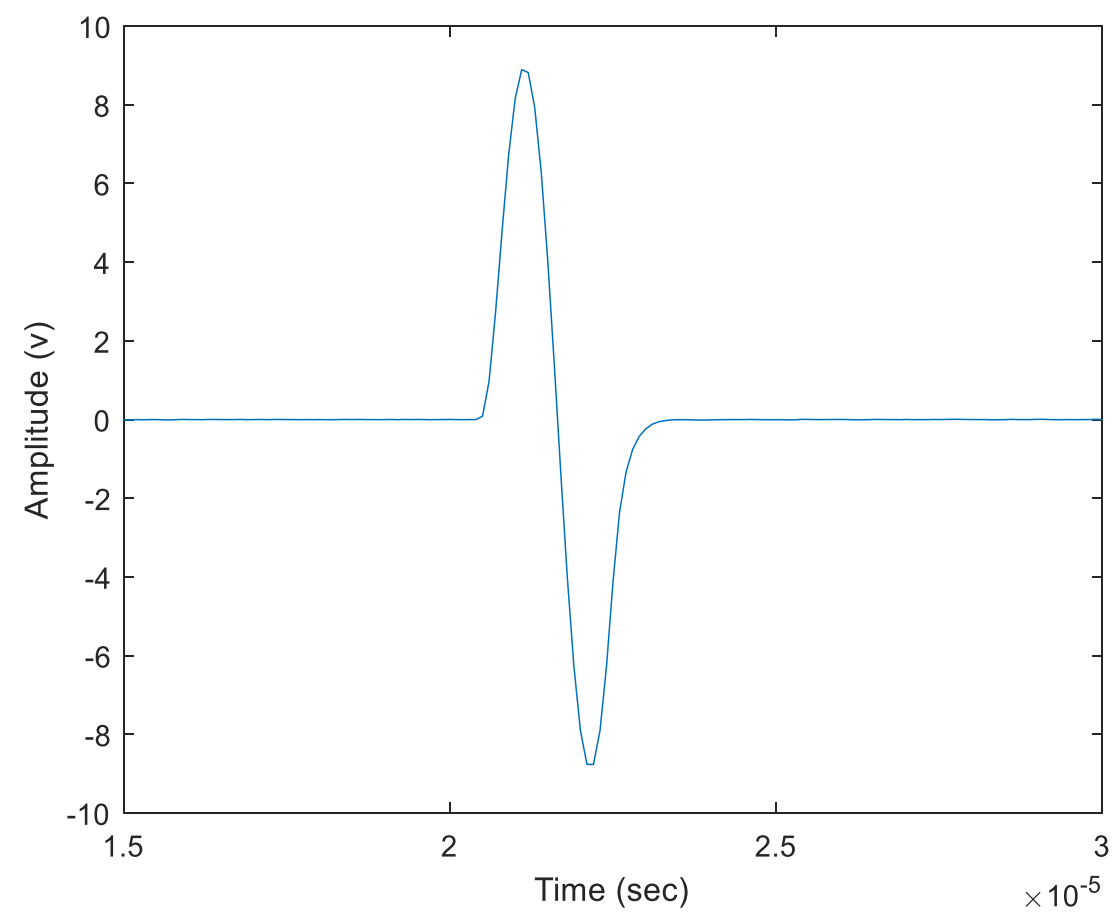

Figure IV-2. Sample of $50 \mathrm{kHz}$ pulse generated by arbitrary waveform generator and sent to transmitting transducer

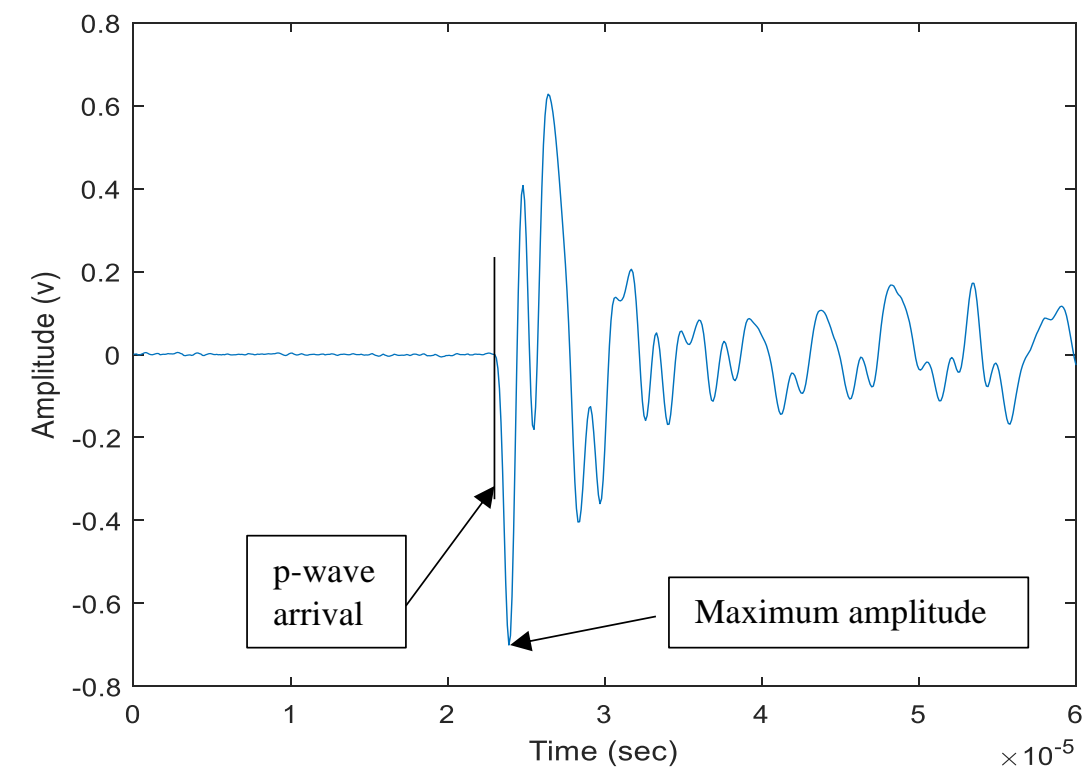

Figure IV-3. Sample of ultrasonic stress wave recorded by receiving transducer.

\section{Test 1: Variable Transducer Coupling Force}

The proposed testing procedure requires the temporary attachment of ultrasonic transducers to a manufactured part. In a mass manufacturing environment, the transducers used 
to test a manufactured part are placed either manually or through a robot. Once a test is concluded, the transducers are decoupled and then coupled again to the next part. This process prohibits the use of couplants such as glue. Rather, the transducers are dry-coupled by pressing them against the part with a certain force.

The objective of this test was to study the effect of the coupling force on the recorded signals. A pulse is transmitted across the steel plate (244 $\mathrm{mm} \times 244 \mathrm{~mm} \times 12 \mathrm{~mm}$ ) and the coupling force is changed to study its effect. Figure IV-5 illustrates the test setup with two applied coupling forces. The rubber bands keep the transducers in position while providing a relatively small normal force to the transducers. At the same time, the rubber bands allow for minimum movement of the transducers (within $2 \mathrm{~mm}$ radius) at each recording.

The recorded ultrasonic reference signal in this study is for a coupling force caused by a $1500 \mathrm{gr}$ steel mass atop both the transducers. The $1500 \mathrm{gr}$ consist of three separate $500 \mathrm{gr}$ masses. Three ultrasonic measurements are performed for four coupling conditions: $1500 \mathrm{gr}, 1000 \mathrm{gr}, 500 \mathrm{gr}$, and no mass (rubber bands only).

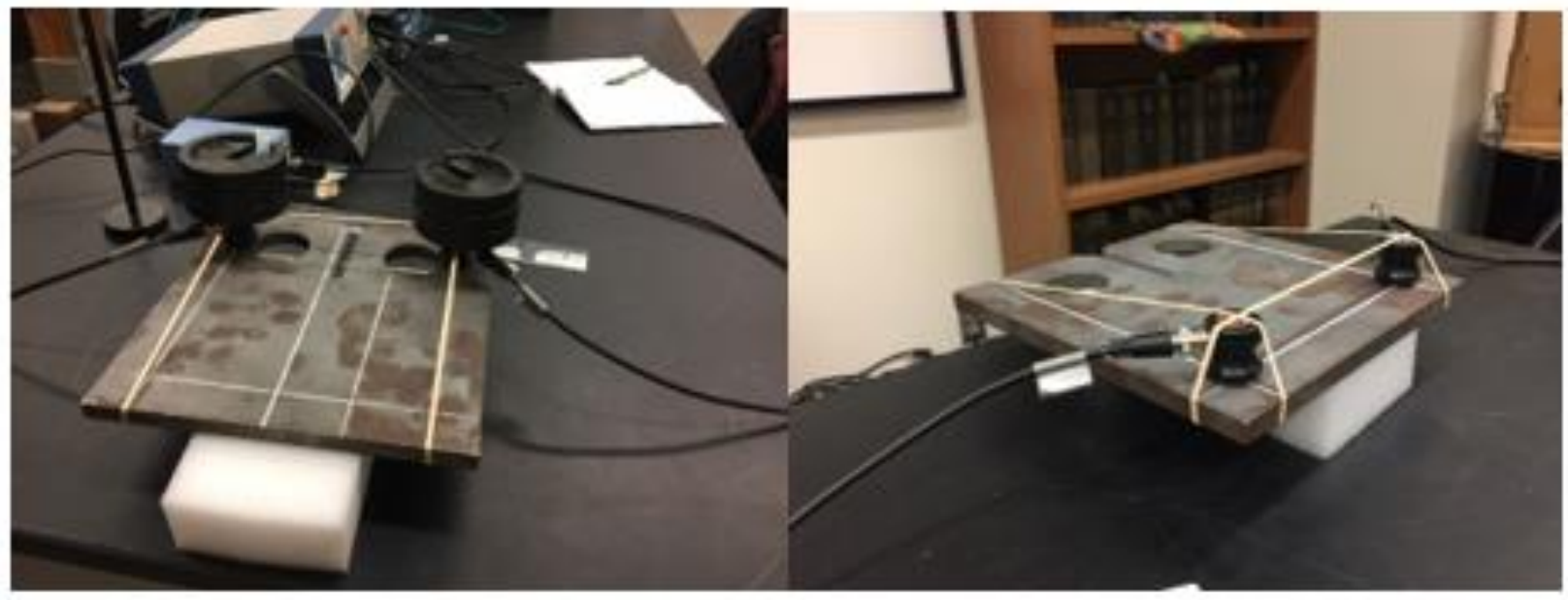

(a)

(b)

Figure IV-4. Images showing steel plate for two select forces: $1500 \mathrm{gr}(\mathrm{a})$ and $0 \mathrm{gr}(\mathrm{b})$ atop the transducers

\section{Test 2: Steel Coupler Nut}

The first sample is a steel coupler nut, as shown in Figure IV-6. The outside is hexagonal shaped with a $25 \mathrm{~mm}$ threaded inside diameter. Each hexagonal side is $20 \mathrm{~mm}$ wide and the total length of the part is $70 \mathrm{~mm}$. This part is used in commercial steel construction and mechanical applications.

A disturbance is introduced to the coupler nut in form of small magnets. The magnets are $12 \mathrm{~mm}$ in diameter and $4 \mathrm{~mm}$ thick. They act as a simulated defect to the nut by adding additional volume and mass to the specimen as they adhere to its surface.

A total of four magnets were placed in eight different configurations, as illustrated in Figure IV6 . For each configuration, five ultrasonic measurements were performed. The transmitted sine- 
pulse had a frequency of $100 \mathrm{kHz}$ and a total of 65,536 samples were recorded, producing waveforms of $6.55 \mathrm{~ms}$ length.

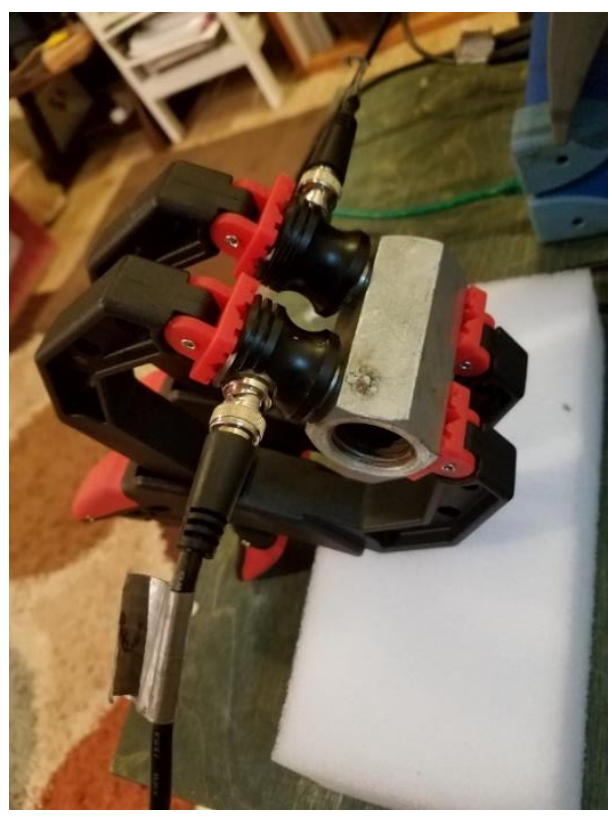

Figure IV-5. Image of coupler nut specimen with coupled transducers

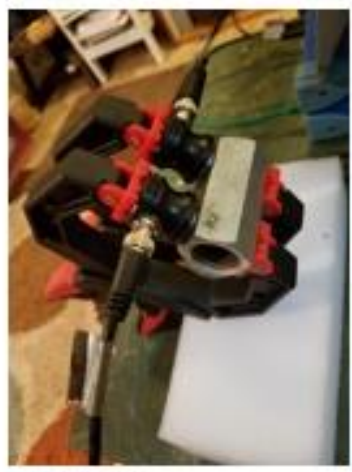

(1) Reference

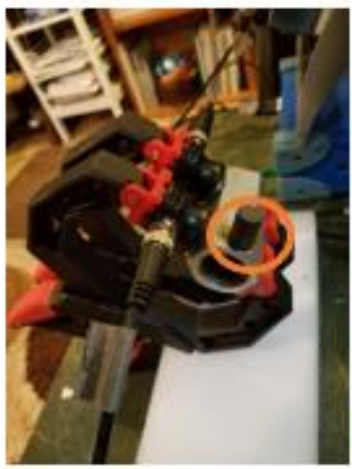

(5) 4 magnets

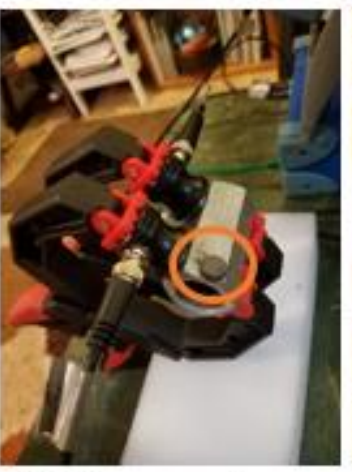

(2) 1 magnet

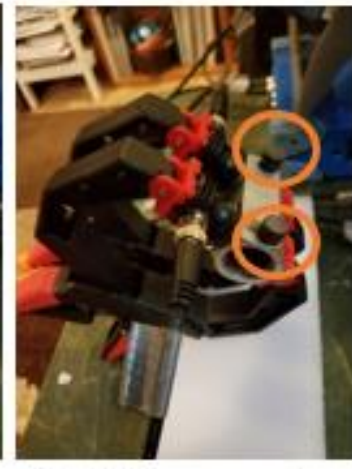

(6) 2+2 magnets

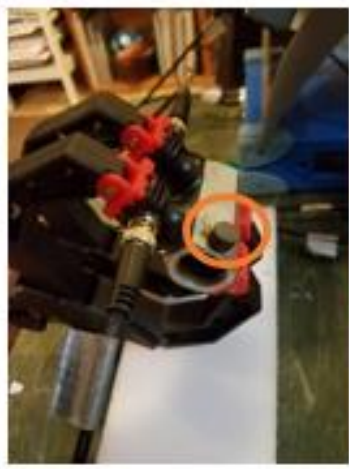

(3) 2 magnets

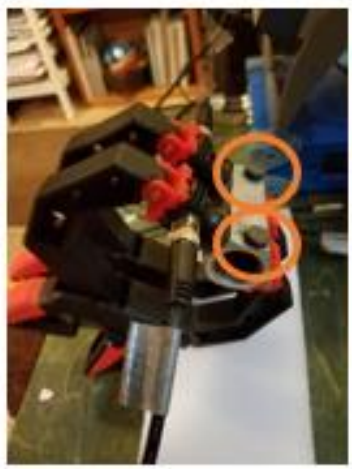

(7) 1+1 magnets

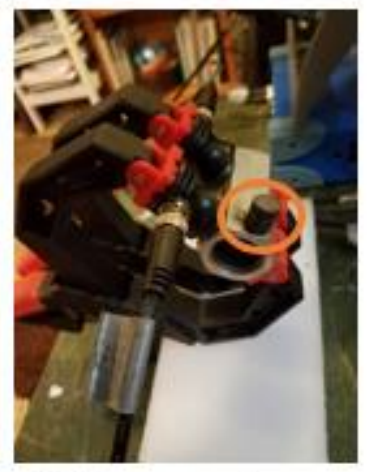

(4) 3 magnets

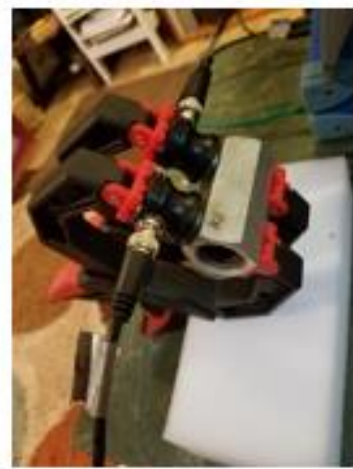

(8) No magnets

Figure IV-6. Image of coupler nut test with different magnet configurations 


\section{Test 3: Steel Plate}

The second test specimen is a flat steel plate with symmetrical manufactured apertures, as shown on Figure IV-8. The plate is $12 \mathrm{~mm}$ thick with a width of $244 \mathrm{~mm}$ and height of $254 \mathrm{~mm}$. Similarly to the coupler nut, magnets are used to simulate the effect of an alteration in the plate. For the plate, the magnets are spread out in different alternating locations of the plate. The plate has a larger surface area, thus allowing for more trialing with the magnets to observe the effects of the distance in the signal recordings.

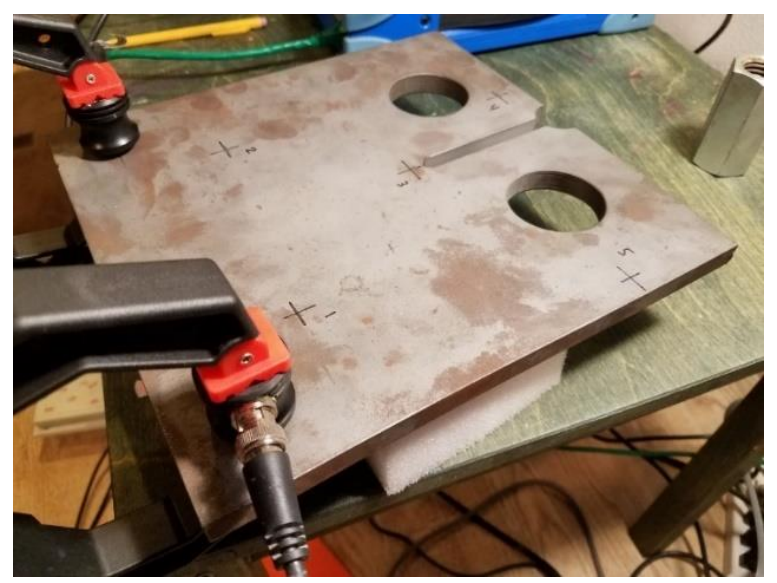

Figure IV-7. Image of steel plate specimen

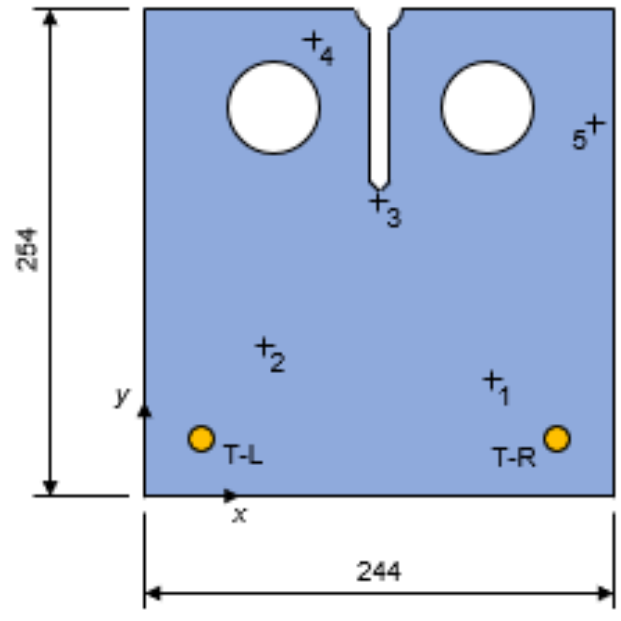

\begin{tabular}{|l|l|l|}
\hline & $x(\mathrm{~mm})$ & $y(\mathrm{~mm})$ \\
\hline T-L & 30 & 30 \\
\hline T-R & 214 & 30 \\
\hline 1 & 181 & 61 \\
\hline 2 & 62 & 78 \\
\hline 3 & 122 & 154 \\
\hline 4 & 88 & 237 \\
\hline 5 & 234 & 194 \\
\hline Plate thickness: $12 \mathrm{~mm}$ &
\end{tabular}

Figure IV-8. Diagram of magnets on steel plate locations

The magnets are attached on the surface of the steel plate. The magnets are not stacked, instead they are placed at different locations of the steel plate, which is shown in Figure IV-9. For each magnet location, the transmitting transducer sends a pulse to the receiving transducer. Remaining at the same locations, the transducers switch tasks, i.e. the receiving transducer becomes the transmitting receiver and vice versa. Thus, the signal travels from left to right in an initial set of magnet positions, subsequently a signal from right to left captures an identical set of magnet 
positions. Ten ultrasonic measurements are performed per magnet configuration. Each magnet configuration consists of a recorded ultrasonic signal of 131,072 samples, at the instant a single magnet is placed at a location between 1 and 5 on the plate.

\section{Test 4: Plastic Edge Triangle}

The third specimen is a straight-edge plastic triangle. The triangles are $203.6 \mathrm{~mm} \times 203.6$ $\mathrm{mm}$ with a thickness of $4.76 \mathrm{~mm}$.

This research obtained three identical copies of the straight-edges, shown in Figures IV-10 to 12. More reproductions of the same specimen allow for more trials of introducing alterations to them. The alterations introduced cut into the material. On the first sample (distributed holes 1), matching $2.36 \mathrm{~mm}$ round holes are drilled into various locations of the triangle. The second sample (distributed holes 2) carries the identical holes in a different order. The third sample (cut holes) receives a series of extended cuts and notches to the edges.

Each alteration generates its own ultrasonic signal recording. Once they are clamped into place, the transducers transmit a stress wave from one to the other after initial clamping and after every notch or hole. Ten recordings of 32,768 samples are taken for every disturbed state of the triangle. The triangle dismounts the clamping system after the end of series of holes and the next straight-edge is put in place. All efforts were made to clamp the specimens in the same location using the same coupling force, in a visual, manually operated procedure. Consequently, the manual procedure cannot guarantee the consistency of the exact location of the transducer placement, nor the force applied by the clamps.

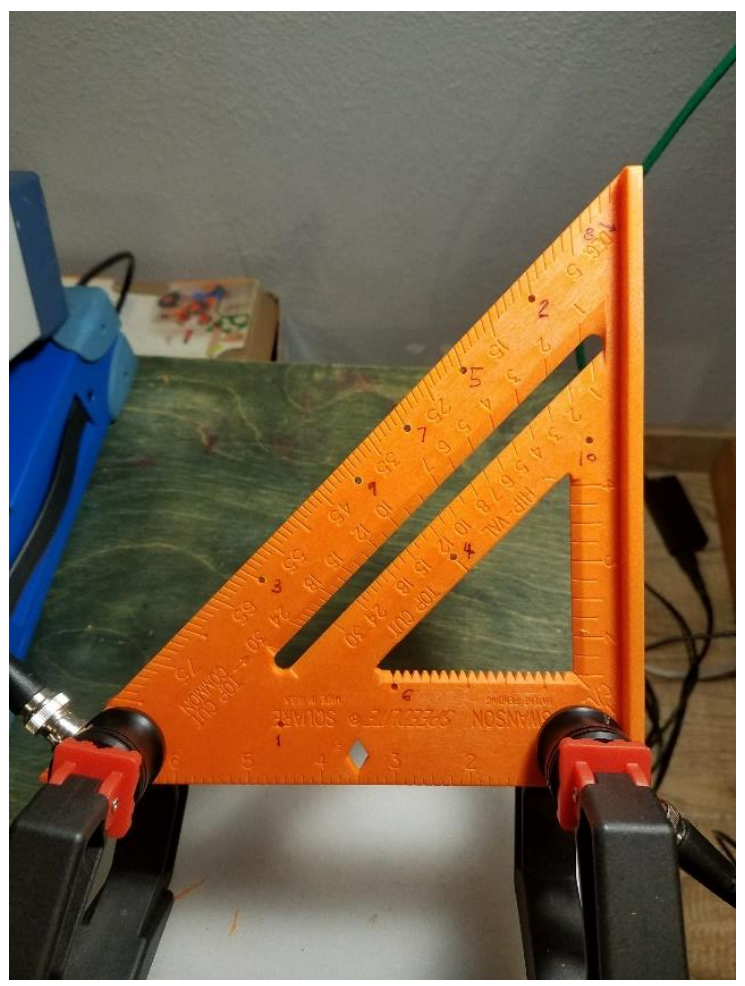

Figure IV-9. Image of straight-edge specimen: distributed holes (1) 


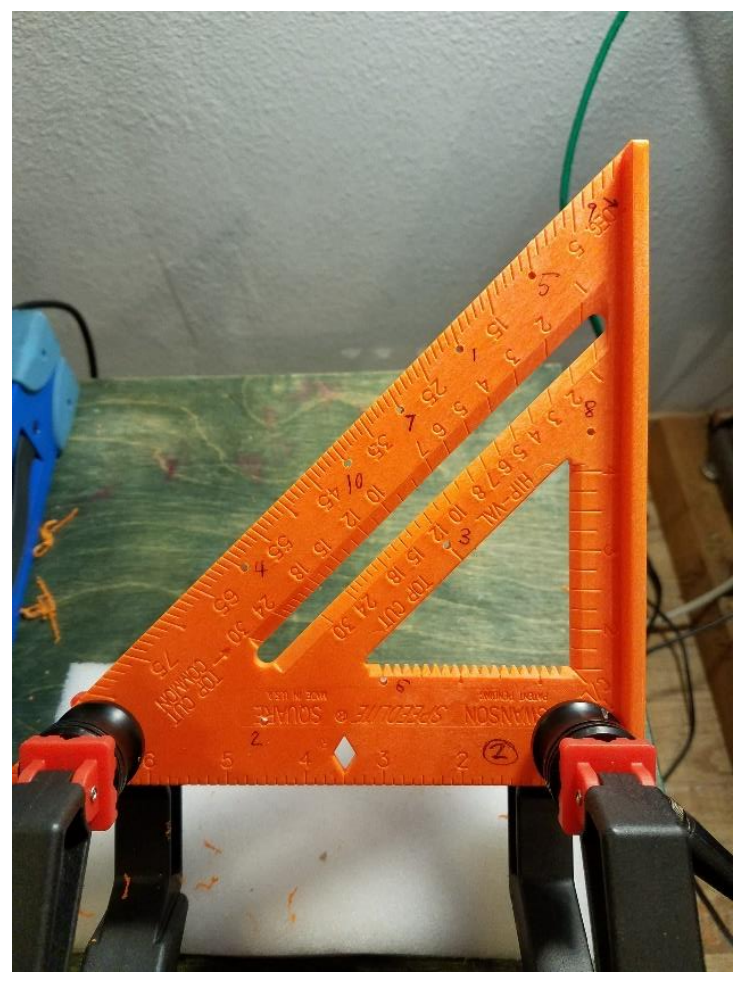

Figure IV-10. Image of straight-edge specimen: distributed holes (2)

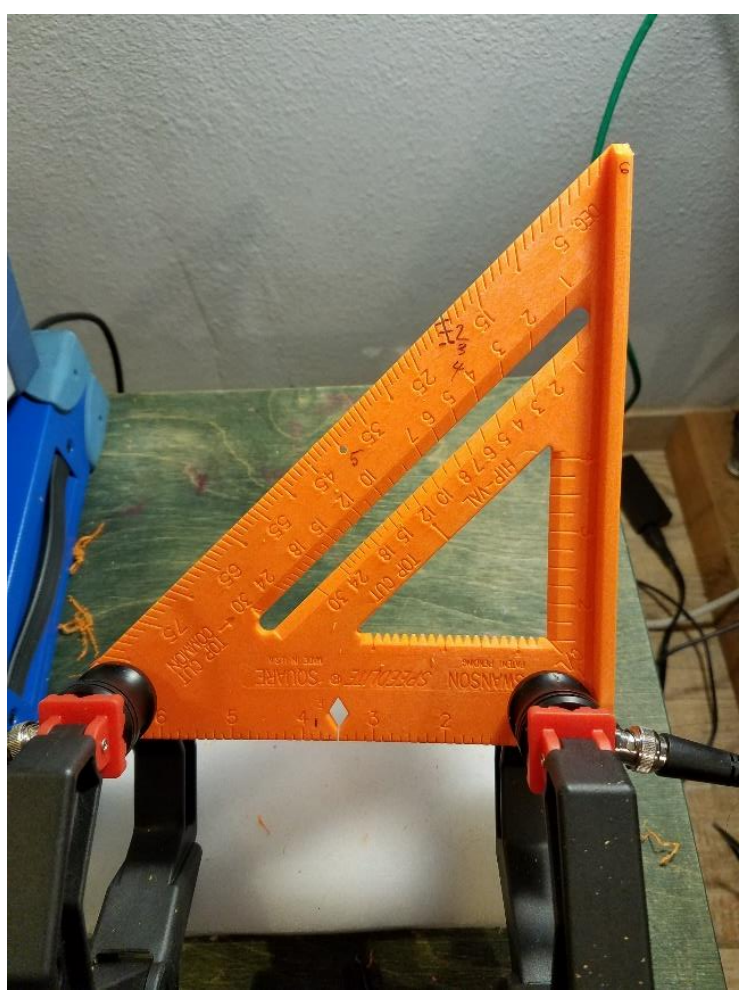

Figure IV-11. Image of straight-edge specimen: cut holes 


\section{Results and Analysis}

\section{Test 1: Variable Transducer Coupling Force}

The objective of this test was to study the effect the applied coupling force has on the recorded ultrasonic signals. The three recorded signals per load case were detrended by subtracting 12000 pre-trigger samples from the entire signal. Then, each detrended signal case was compared with each of the three reference (i.e., $1500 \mathrm{gr}$ ) load case signals by calculating the coefficient of determination, $R^{2}$ between the signals' amplitudes. For the reference case, the three detrended reference recordings were compared to each other. A plot of $R^{2}$ values vs. coupling force is provided in Figure V-1.

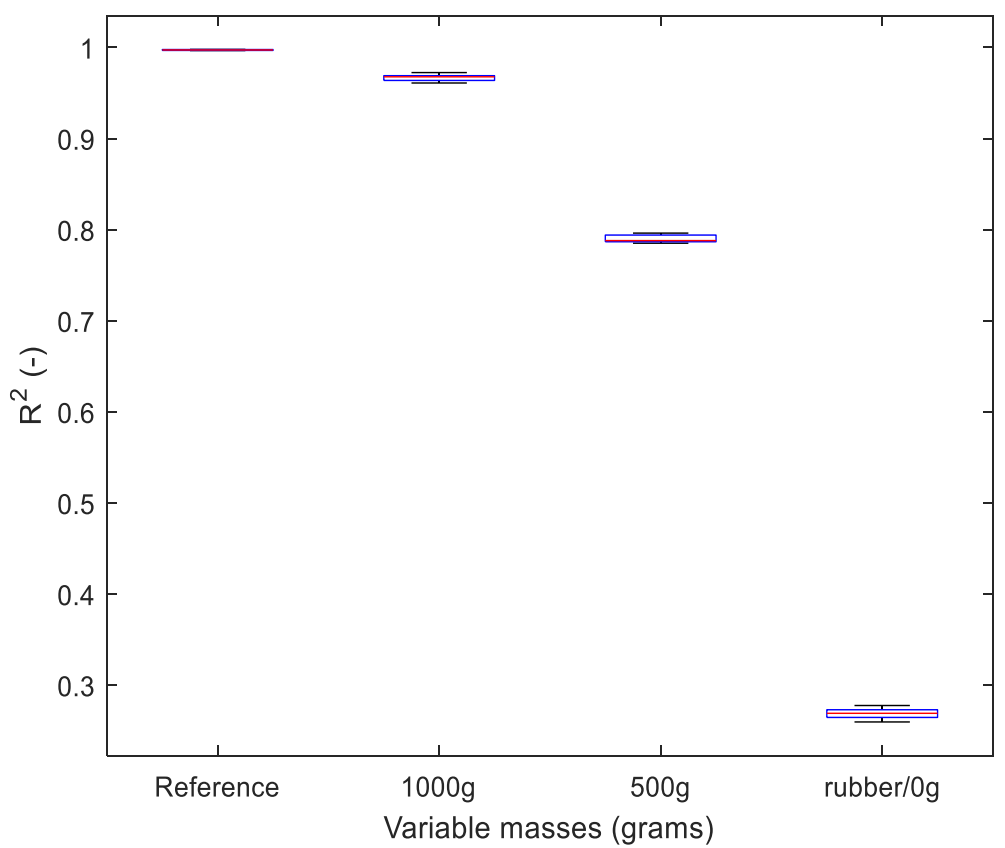

Figure V-1. Plot of $R^{2} v$ s. coupling force. Error bars represent the minimum and maximum of $R^{2}$

One observation is the consistency of signal recordings within the same case. For example, for the reference case (i.e., 1500 gr mass), the reference signals compared to each other produce a coefficient of determination, $R^{2}$ of approximately 1 . This means that the noise level between measurements of the same case, i.e. where the coupling conditions are constant, is negligible.

As can also be observed, a change in the coupling force has a significant impact on the recorded signals: the $R^{2}$ decreases with decreasing coupling force. This sensitivity highlights the critical requirement for coupling force consistency for the proposed measurement approach.

\section{Test 2: Steel Coupler Nut}

The objective of this test was to observe the change in the recorded ultrasonic signals as magnets were added to the steel coupler nut. An ultrasonic signal was recorded each time a magnet was placed at different locations of the part. Five ultrasonic measurements were performed for each magnet configuration. Signals were detrended by subtracting the signal 
average over 3032 samples. Then, the five recorded signals per case were stacked (i.e., added together). Subsequently, each stacked signal was compared with the reference case by calculating the coefficient of determination, $R^{2}$.

\section{Signal Partitioning to Determine Sensitivity}

First, a study to determine which portion of the signals was most sensitive to the introduced alterations was performed. For each test case, including both the magnet and reference cases, signals were partitioned into different numbers of equal-length segments, referred to as "windows" in this test. The number of windows, $n$ was in terms of $2^{x}$, where $\mathrm{x}$ ranged from 0 to 5 , leading to eight analyses.

For each $n$, the $R^{2}$ was determined for each window. The graphs in Figure V-2 show both $R^{2}$ vs. test case as well as which portion of the signal exhibited the strongest sensitivity.

The plots show how the $R^{2}$ coefficient changes as the signals are partitioned into smaller windows. When the signal is taken in its entirety, the coefficient of determination does not vary significantly, indicating low sensitivity. The sensitivity increases notably once the signal is subdivided, with the later portions being more sensitive. The increasing number of windows highlights the portion with the lowest correlation to the reference, which is consistently the latter portion wave of the signal, the coda wave. The only exception is for $n=32$, which is when the windows become so short that the first window only contains noise. Naturally, this will produce the lowest $R^{2}$, but can easily be dismissed.

\section{Down-Sampling of Signals}

Second, the effect of sampling frequency on the ability of the coda wave to capture alterations was studied. For this analysis, a fixed number of windows, $n=4$ was selected.

At first, the signals compare to each other across the entire number of samples provided by the high-speed data recorder. The maximum sampling rate of $10 \mathrm{MHz}$ was limited by the high-speed recorder, in this case providing 65,536 samples. Next, MATLAB was used to decrease the sampling rate of the signals, hence reducing the number of samples per signal recording. The sampling rate was decreased by a factor of the power of two.

The determination coefficient remains remarkably consistent across all sampling rates. As the sampling rate decreases, the trend of the coefficient for each "alteration" remains similar to the trend from the full $10 \mathrm{MHz}$ signal. The trend starts to lose similarity around the $20 \mathrm{kHz}$ signals, at which point it is significantly lower than the central frequency of the transmitted $100 \mathrm{kHz}$ sine pulse. This demonstrates the proposed approach's potential for detecting alterations even with low frequency equipment.

Finally, it can be observed that, like for the previous test, it is always the last of the four windows that appears to have the highest sensitivity to alterations in the part, again demonstrating the capabilities of coda wave analysis. 

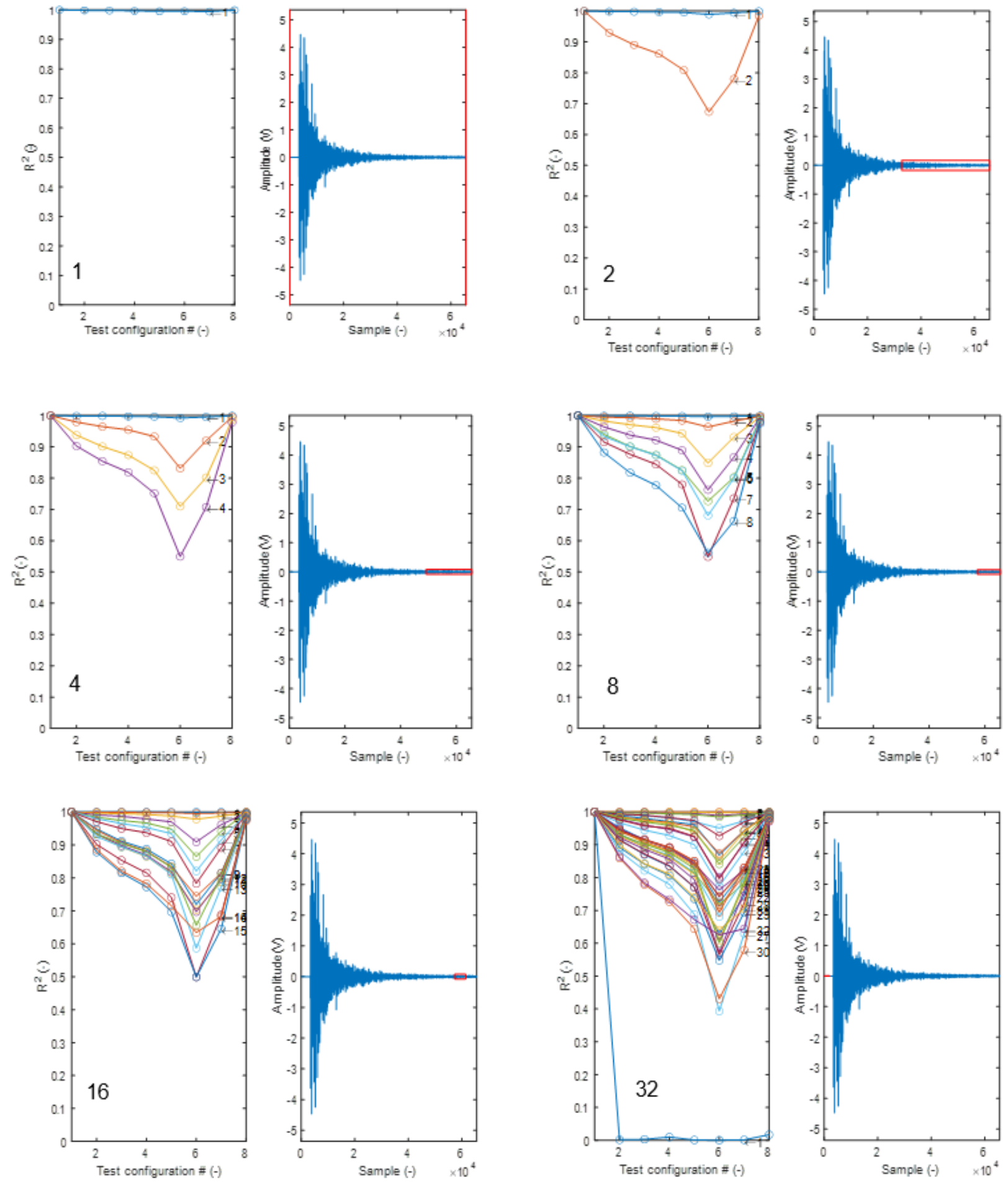

Figure V-2. Comparison of $R^{2}$ for different numbers of windows (indicated by number in lower left corner of left figures) used to subdivide signals. Right figures show a sample signal in the time domain with a red box indicating the window with the signal portion exhibiting the highest sensitivity. 

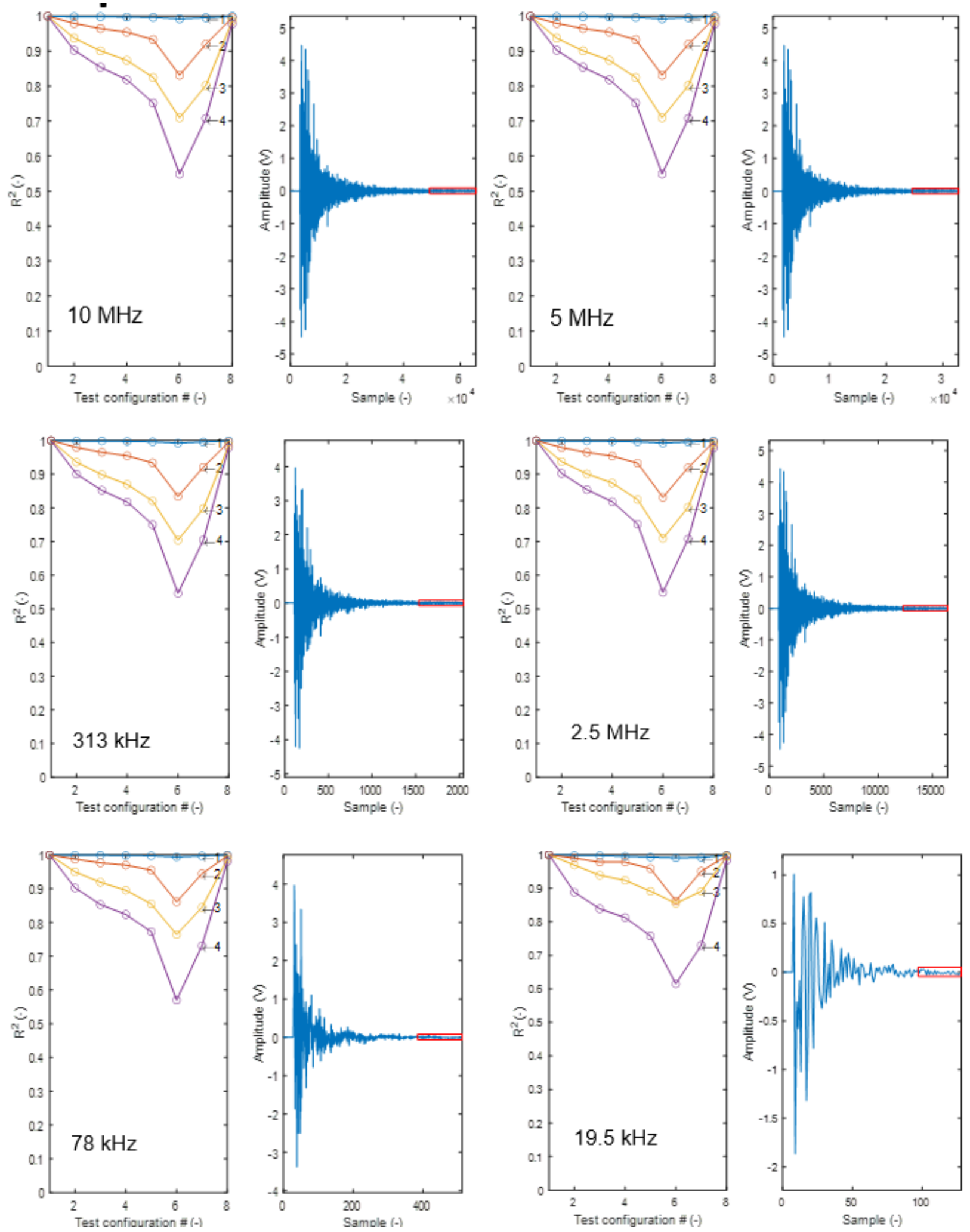

Figure V-3. Comparison of $R^{2}$ for different sampling rates (indicated by number in lower corner of left figures) used to subdivide signals. Right figures show a sample signal in the time domain with a red box indicating the window with the signal portion having the highest sensitivity. 


\section{Sliding Window Correlation Analysis}

In this analysis, instead of correlating two signals or fixed-location widows of two signals, a sliding window of fixed length was employed. The window, with a 3032 sample length, is dragged across the entirety of the two signals. The $R^{2}$ is calculated at each time increment across the window. The resulting array of values show the progression of the coefficient of determination across the signals and is shown in Figure V-4.

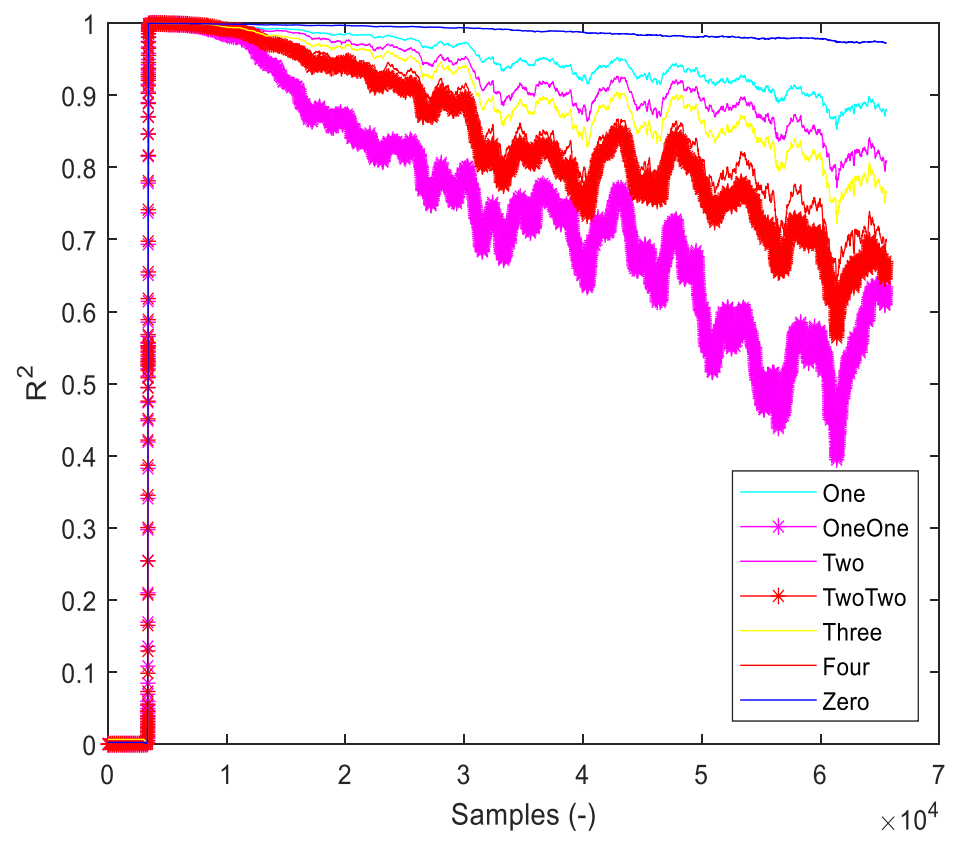

Figure V-4. Plot of $R 2$ sliding window analysis for all eight magnet configurations.

The coefficient of determination clearly distinguishes the "altered" cases from the "reference" case. As early as the first third portion of the signal, the $R^{2}$ for the signals featuring magnets (denoted "One" through "Four" on Figure V-4) separates itself from the case when all magnets were removed again (denoted "Zero" on Figure V-4). At the end of the sliding window, the correlation between "altered" and "reference" does not surpass a correlation of 0.9 , clearly distinctive from the reference signal measurements between each other.

At the same time, all the cases with alterations share notable characteristics in their $R^{2}$ sliding window analysis. The seven cases with magnets exhibit shared peaks and dips in their slope, regardless of how far apart they are from the reference case. The dips can be interpreted as locations in the signal that are particularly sensitive to the introduced alterations.

Interestingly, the greatest differences in $R^{2}$ do not arise from the highest quantity of added magnets, but from their location. The plot lines differing the most come from the cases where there is a magnet located close by the transmitting transducer ("One-One" and "Two-Two"). It is speculated that for these cases, the magnets impact the diffuse waves from an earlier point of the signal, thus creating greater discrepancy over its entirety. 


\section{Test 3: Steel Plate}

\section{$P$-Wave Speed}

To determine the P-wave speed of the steel plate material, the two transducers were placed directly across from each other on either side of the steel plate, as is shown in Figure V-5.

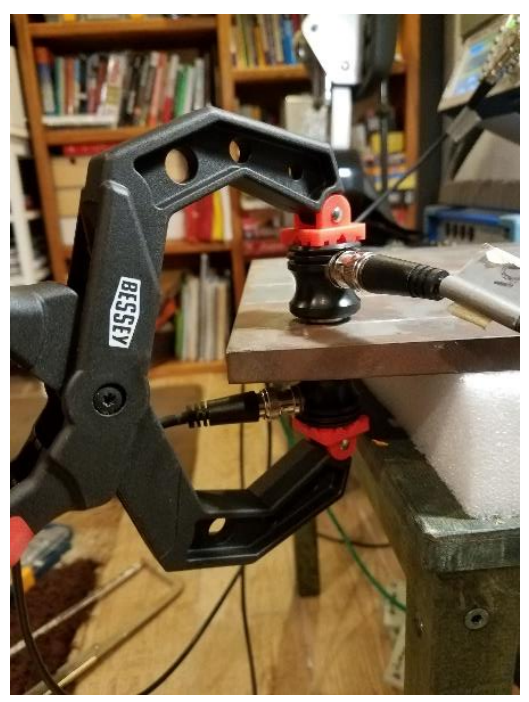

Figure V-5. Image showing p-wave speed setup

The wave speed is determined by dividing the thickness of the steel plate by the time-of-flight (TOF) of the P-wave. The TOF is established by subtracting the departure time of the transmitted wave from the arrival time of the P-wave arrival, also referred to as onset, in the recorded signal. Both departure and arrival times are obtained numerically by using a picking algorithm. The algorithm chooses the time corresponding to the moment the signal surpasses an amplitude threshold. The resulting P-wave speed was on average $4926 \mathrm{~m} / \mathrm{s}$ across five measurements, slightly below the reference value of a stress wave speed in steel materials typically reported [21].

The P-wave speed was used in the subsequent analysis. 


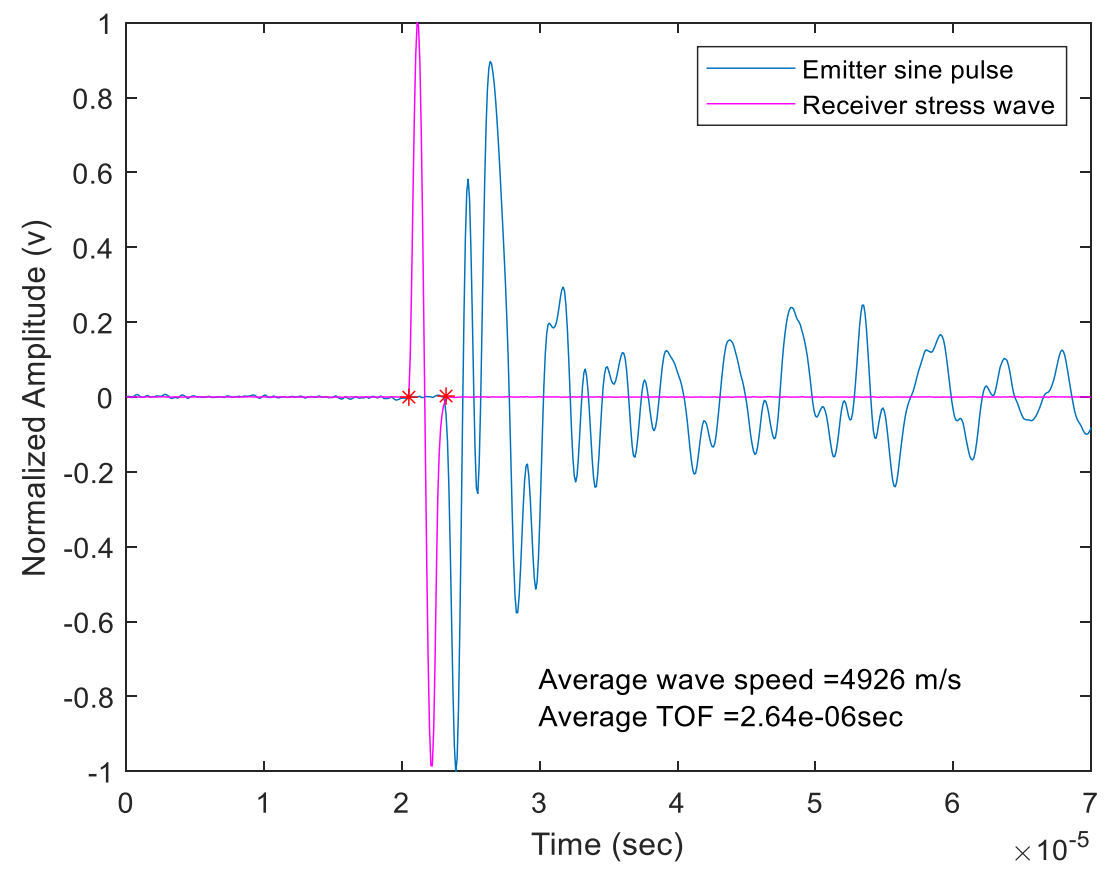

Figure V-6. Illustration of p-wave speed test: amplitude vs time of transmitted pulse (magenta) and recorded signal (blue). The red '*' indicates the onset of the transmitted pulse and the $p$-wave arrival time of the recorded signal.

\section{Defect Localization from Sliding Window Analysis}

For this test setup, the stress wave propagates throughout the part, arriving and interacting at an "alteration" on the plate at a specific time. Based on this principle, the wave speed and travel distance can be used to determine when changes in the recorded signal can be expected. In other words, the ultrasonic signature of an "altered" test case should start to differentiate from the reference case at the time moment it arrives at an alteration (here a magnet).

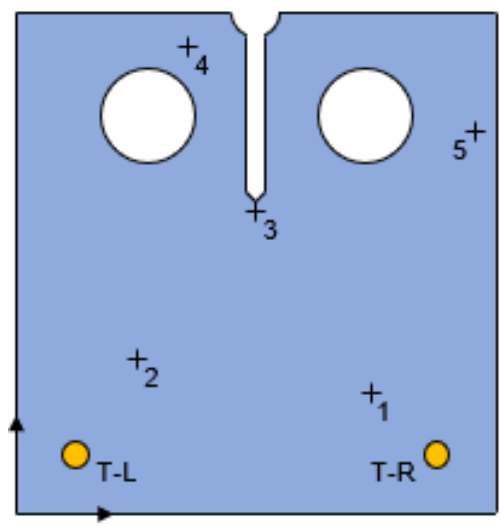

Figure V-7. Illustration of magnet locations on steel plate (for coordinates see Figure IV 8) 

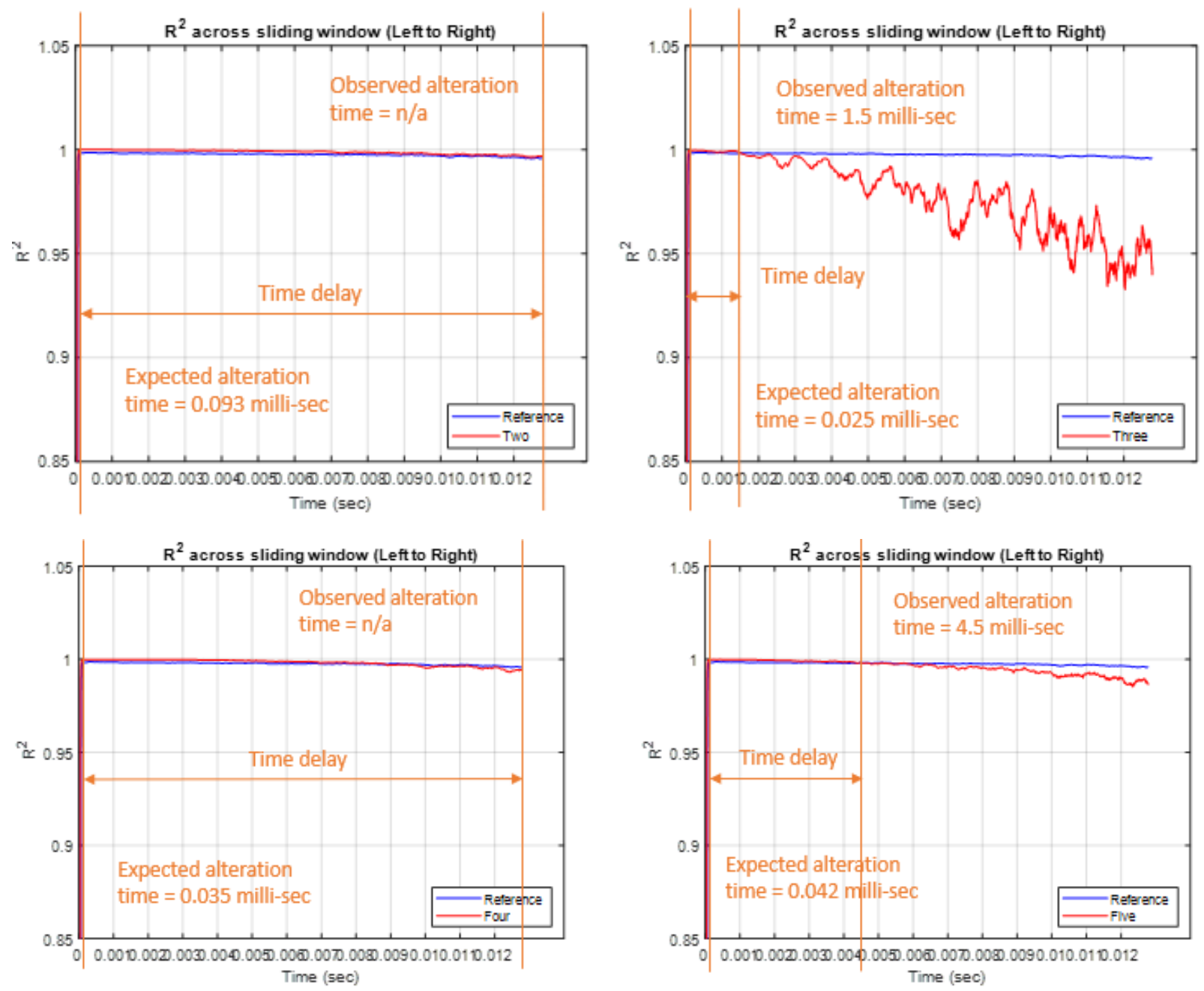

Figure V-8. Comparison of R2 sliding window analysis for magnet positions 2 through 5 on the steel plate with stress wave traveling from left transmitter to right receiver transducer. The expected and observed time for alteration arrival (in orange) show the delay of the coda wave to capture the alteration.
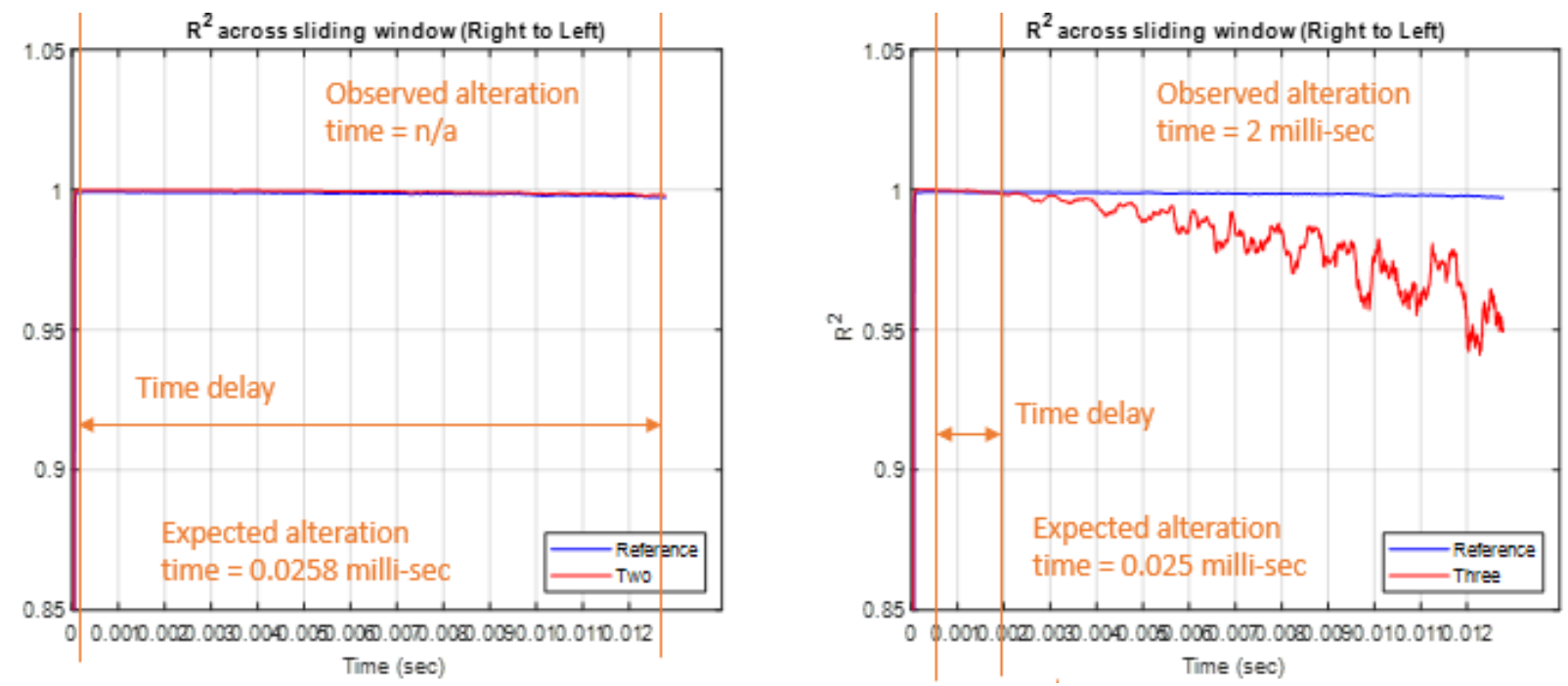

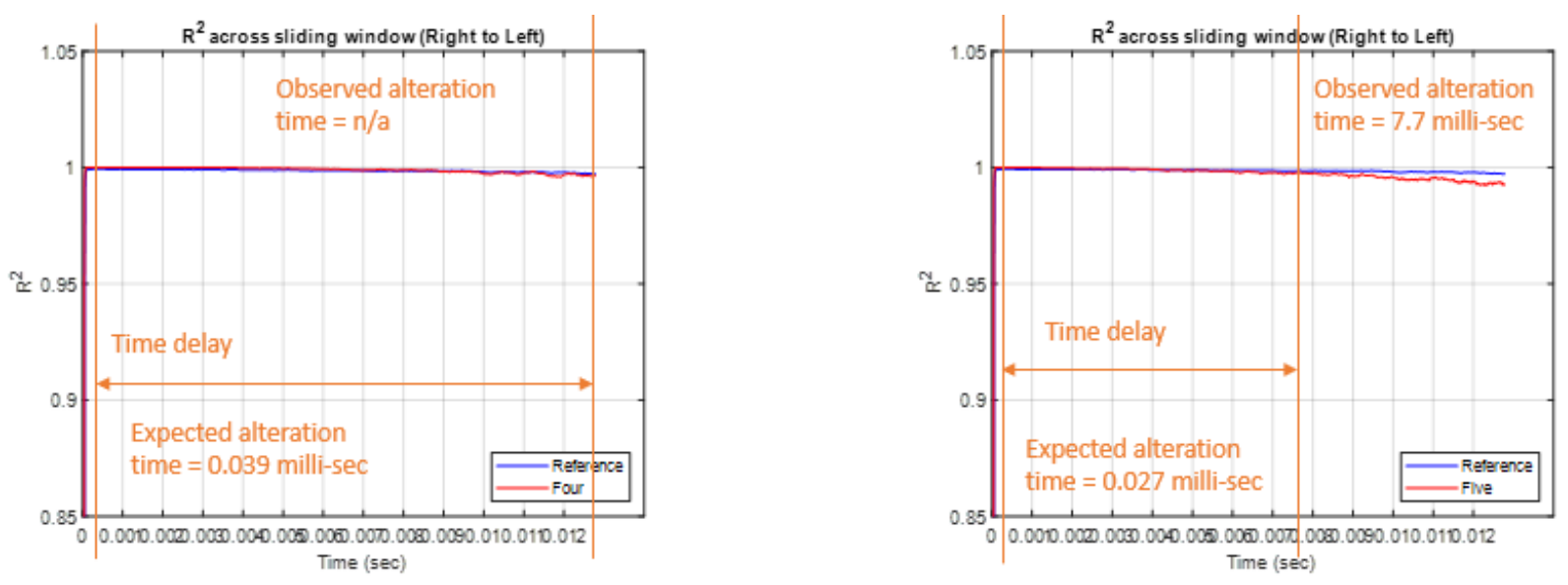

Figure V-9. Comparison of R2 sliding window analysis for magnet positions 2 through 5 on the steel plate with stress wave traveling from right transmitter to left receiver transducer. The expected and observed time for alteration arrival (in orange) show the delay of the coda wave to capture the alteration.

The alterations are more difficult to observe in the steel plate specimen. As a start, the coefficient of determination sliding window does not recognize between the reference and certain magnet positions, such as position 2 and 4 in the left to right case and the right to left case (magnet positions are shown on Figure V-7). Also, the range of difference in the $R^{2}$ value is smaller. In the cases where the magnets are recognizable, the coefficient does not decrease below 0.9. Thus, it is less distinguishable to read the altered cases on the steel plate.

Furthermore, the time delay to recognize the alteration does not correspond to the stress wave travel time. Figure V-8 and 9 illustrate the expected moment in time for the wave to arrive at the alteration, which is calculated from the distance between magnet and emitter and the wave speed. Equally, it draws the moment where the correlation comparison starts to deviate from the reference signature. The observed defective moment carries a time delay between 2.5 and $8.0 \mathrm{~ms}$ after the wave is supposed to capture the defect. Hence, the direct observation of the alteration arrival does not yield the location of the magnet.

Nonetheless, the magnet locations cause a significant impact in the ultrasonic signature of the recording. For magnet locations 3 and 5 , the $R^{2}$ comparison is distinctive to the reference recording, both in the left-to-right and right-to-left cases. These locations generate a significant impact in the coda wave portion between the two transducers. The location of an identical magnet on the plate determines the degree of distinction based on the $R^{2}$ measure.

\section{Test 4: Plastic Straight-Edge Triangle}

\section{Coefficient of Determination Limitations}

Initially, this study performed the $R^{2}$ sliding window analysis based on the coefficient of determination to compare the altered signals from the straight-edge triangles. The signal processing methodology proceeded identically to the coupler-nut and steel plate specimens. The signals from "reference" and "altered" cases in the distributed holes (1) specimen were first detrended and then stacked. Then, a window of determined length was swept across the entire duration of the signals, calculating the correlation coefficient between the two signals at each step. The array of $R^{2}$ values at each position are plotted against time in Figure V-10. 


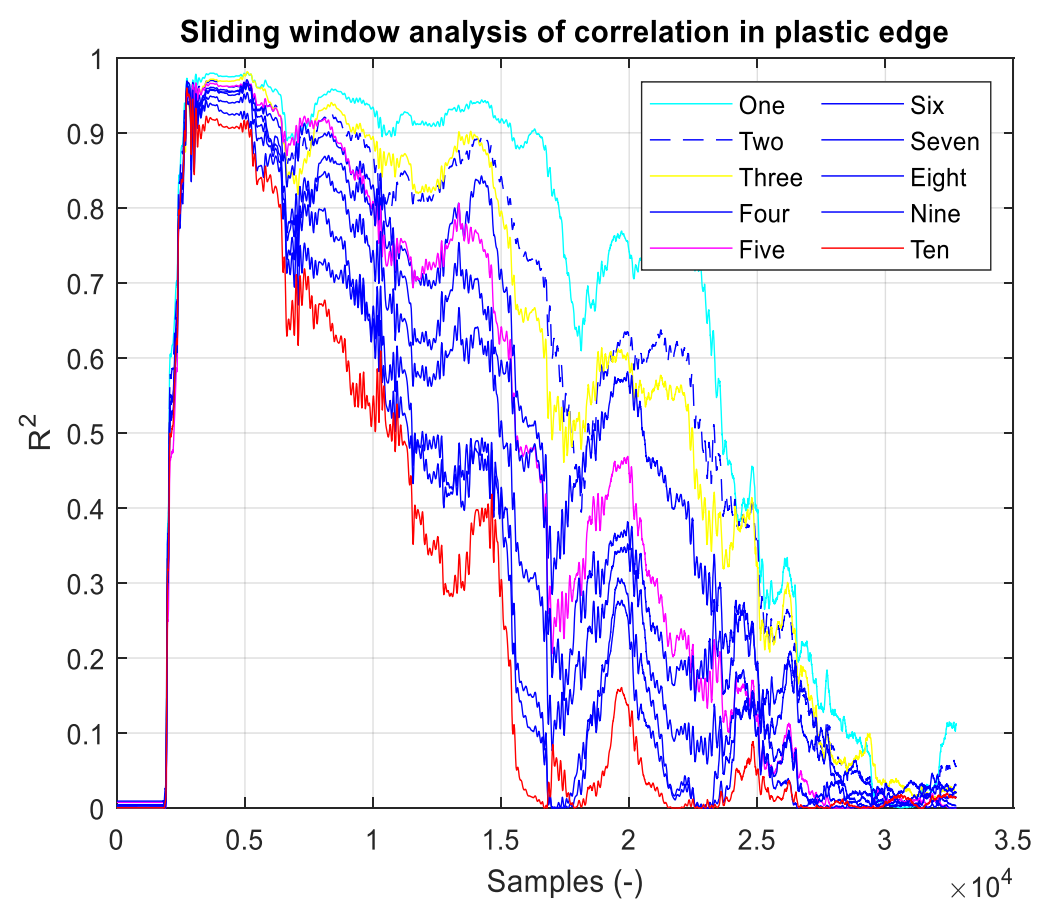

Figure V-10. Plot of $R 2$ sliding window analysis for all ten perforation cases in straight-edge distributed holes (1) specimen.

Overall, it appears that the $R^{2}$ value on the straight-edge specimens is significantly more sensitive to introduced alterations. All of the ten alteration cases decrease quickly in correlation to the reference, at an early segment portion of the coda wave. Equally, the comparison reaches a zero threshold by the end of the recording, sometimes as early as midway in the wave. The high sensitivity translates to a rougher distinction between the various "altered" scenarios. This further highlights the importance of selecting an appropriate window for the coda wave analysis, which is application dependent.

In addition, the sliding window evaluation is subject to the synchronicity between the signals. In the distributed holes (1) scenario, the reference recording was found to arrive at a slightly earlier time instant to the receiver than the altered cases recordings. This delay causes a decorrelation in the $R^{2}$ that is (a) unrelated to any alterations and (b) indistinguishable from them.

This delay was corrected by padding (resulting in a time shift) of either the reference or altered signals. The amount of padding necessary was determined manually but can also be found through numerical processing, e.g. by using cross-correlation.

\section{MSC Implications and Benefits}

In order to resolve the previous issue, the MSC estimate was used to determine the similarity of two recorded ultrasonic signals. MATLAB was employed to compute the coherence of the "altered" and "reference" signals as a function of frequency. Both "altered and "reference" signals were previously detrended and stacked.

The range of frequencies of the MSC function extends from zero to the Nyquist rate of the recorded signal, which in these tests was $5 \mathrm{MHz}$. Over the range of frequencies shown in Figure 
$\mathrm{V}-11(\mathrm{a})$, the MSC function shows some correspondence at the beginning portion between the reference signals. It is expected for the two reference signals to produce an MSC value of near 1.0 , and this is the case from the start until it nears the $100 \mathrm{kHz}$ value of the MSC graph, visible in Figure V-11 (b). This range hovers around the frequency belonging to the transmitted stress wave, in this case a $50 \mathrm{kHz}$ sine pulse (indicated by red dashed vertical line). The highest coherence between the two signals is on either side of the Nyquist rate of the sine pulse. The strong decorrelation for frequencies higher than approximately $90 \mathrm{kHz}$ is because there is no actual signal content.

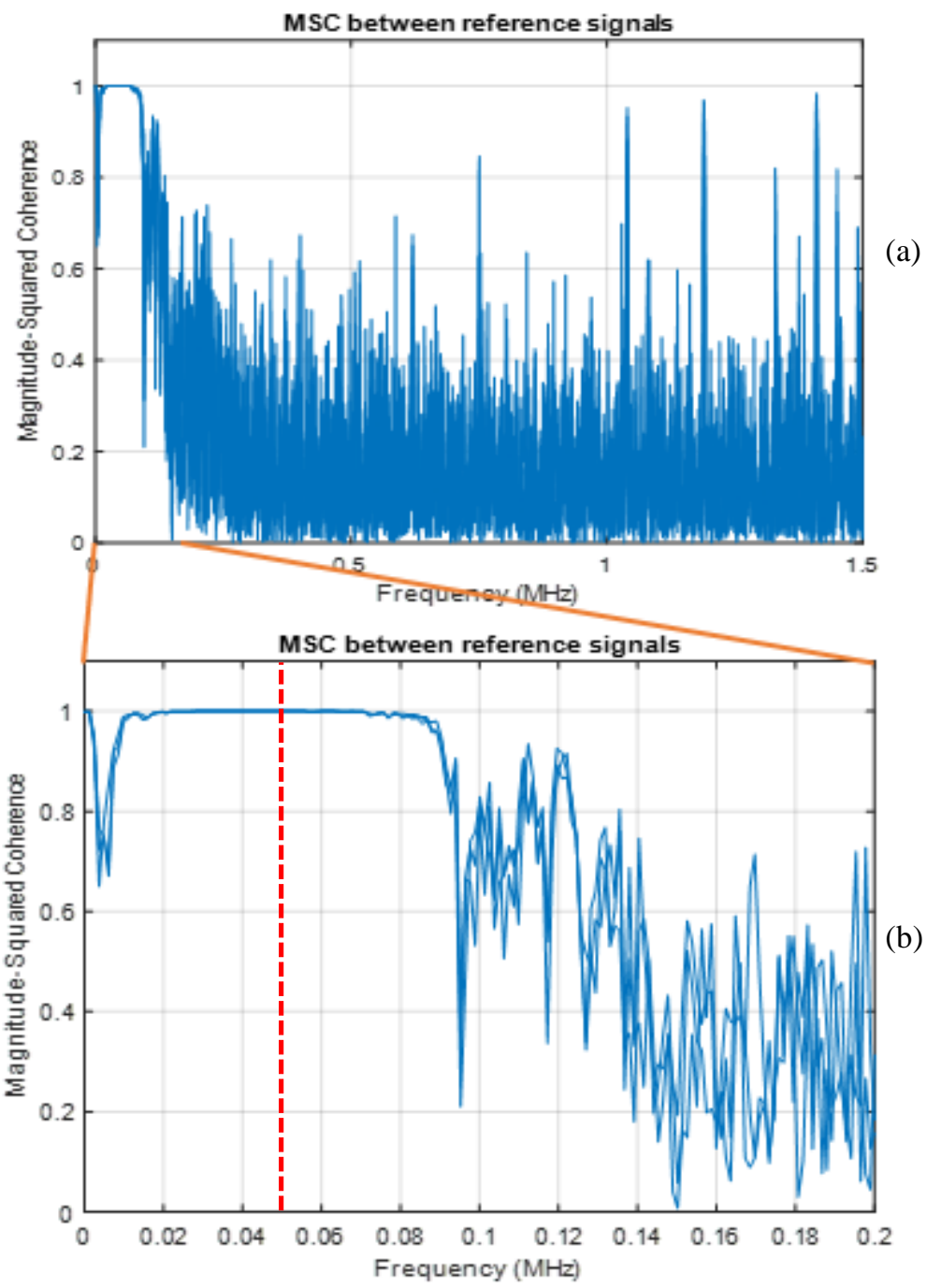

Figure V-11. MSC estimate between two signal recordings of a straight-edge specimen in its' "reference" case. MSC evaluation is from (a) 0 to $1.5 \mathrm{MHz}$ and (b) 0 to $200 \mathrm{kHz}$. The red dashed vertical line indicates the central frequency of the transmitted pulse.

As mentioned earlier, the MSC is insensitive to time shifts between two signals, since the analysis is done in the frequency domain. Hence, differences between arrival times of the signals do not influence the coherence observed by the MSC measure. This might also be a benefit for dealing with differences in sensor coupling. 


\section{MSC Integral Analysis}

In order to produce a single value, MSC values are integrated across a meaningful range, and dividing the integral by the number of samples in the interval. The resulting similarity index has ranges between 1.0 (full coherence) and 0 (no coherence) and can thus interpreted in the same manner as $R^{2}$.

The interval selected for these tests was 20 to $80 \mathrm{kHz}$. Between these values, the MSC measure is expected to be most sensitive to the introduced alterations, between the triangle with one holes and the triangle with ten holes. The MSC integral for one perforation, shown on Figure V-12 (a) shows more coherence with the reference signal, as expected it just below the 1.0 value (MSC integral = 0.99). In contrast, the ten perforations case, shown in Figure V-12 (b) has an MSC integral varying between 0.9 and 0.7 (MSC integral $=0.96$ ). After the $80 \mathrm{kHz}$ threshold, the signal comparison starts to decrease in both cases. Outside of this threshold, the MSC estimate is correlating the noise content of the two signals.
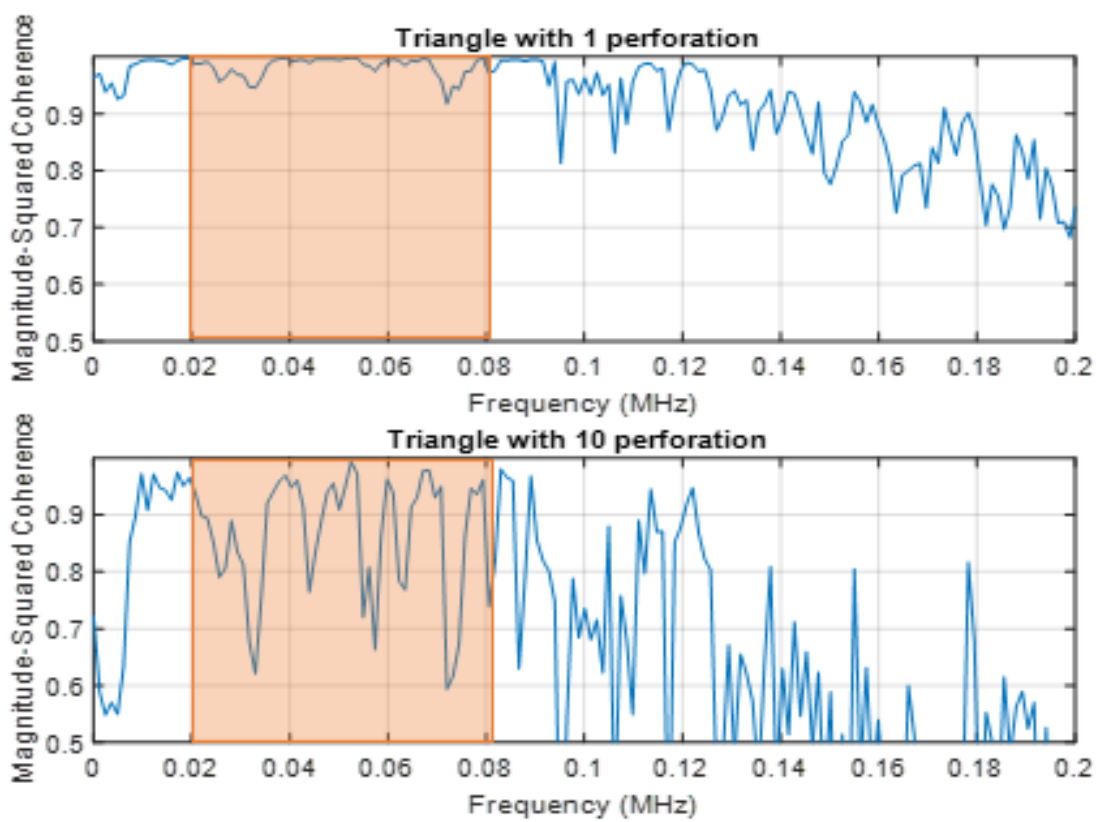

Figure V-12. Plots of MSC estimate values for straight-edge specimen distributed holes (1) with (a) one perforation and (b) ten perforations.

The MSC integral illustrates the decrease in similarity as the straight-edge specimens experience increasing number of deformations. For each straight-edge, the MSC integral for each test case of alteration vs. the reference signals was computed. The MSC integral clearly decreases with increasing alterations (i.e. number of holes). The MSC value reduces to 0.99-0.98 at the first perforation in all three triangles. For distributed holes (1) on Figure V-13, ten perforations cause a reduction to 0.96 , in comparison to 0.86 for distributed holes (2) on Figure V-14. In the cut holes specimen shown in Figure V-15, the decrease reaches 0.975 for six notches. Therefore, it is possible to distinguish between an altered triangle from the comparison to a reference signal using the MSC integral. 


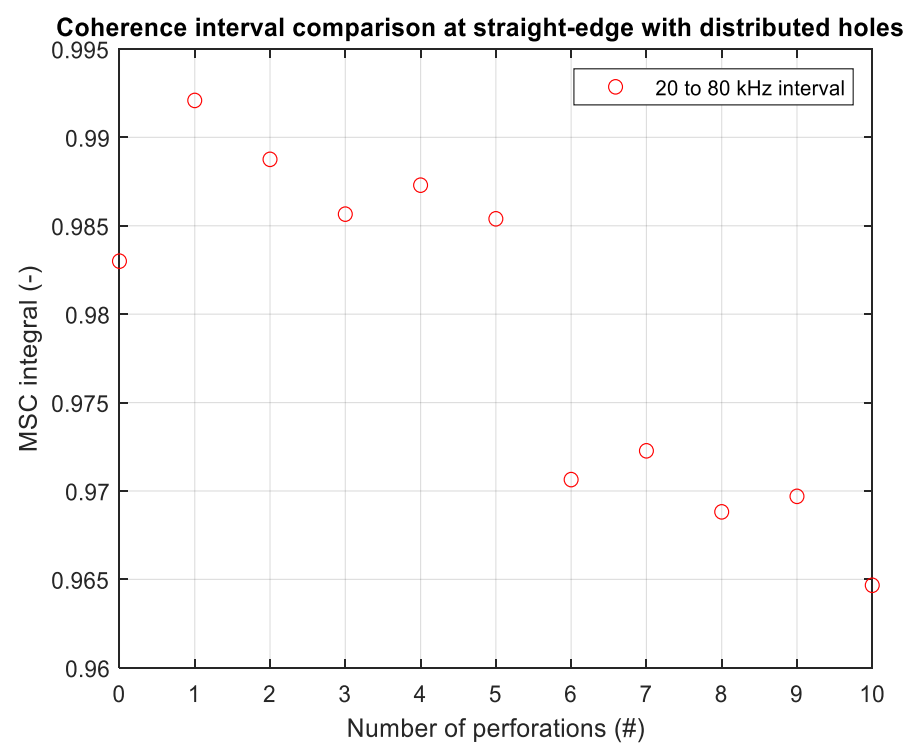

Figure V-13. MSC integral values for all perforation states on distributed holes (1) specimen.

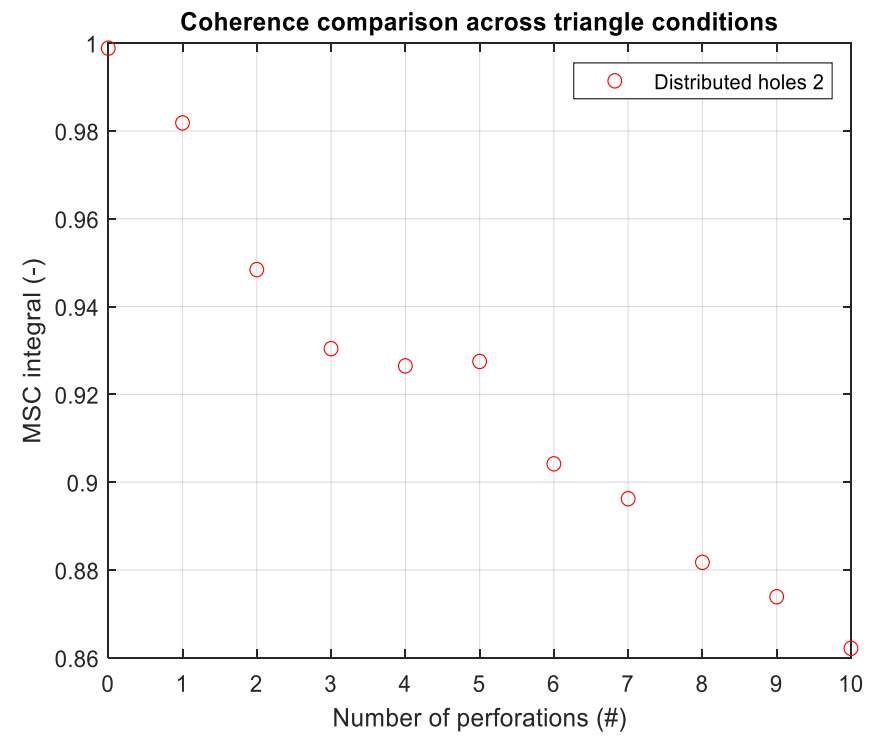

Figure V-14. MSC integral values for all perforation states on distributed holes (2) specimen. 


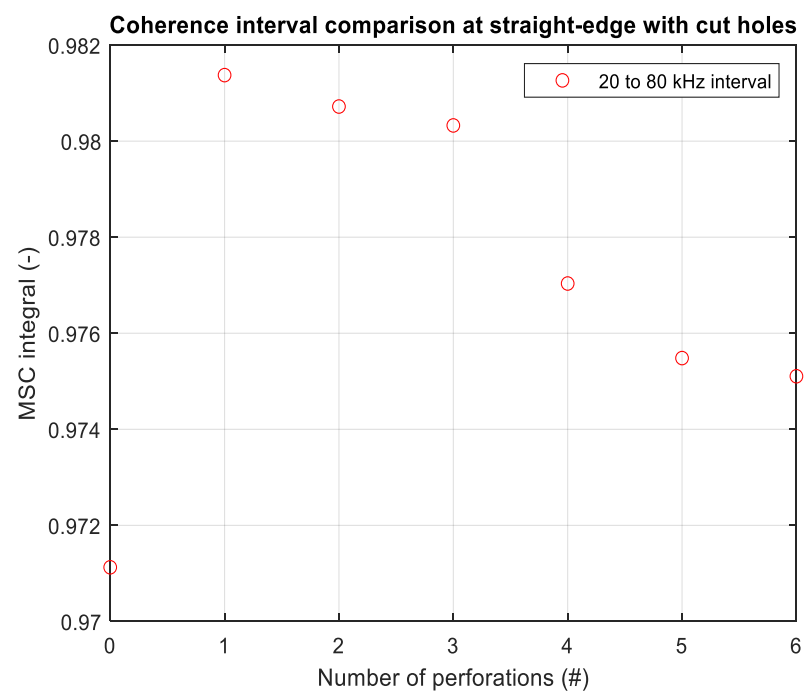

Figure V-15. MSC integral values for all perforation states on cut holes specimen.

\section{MSC Slice Study}

As demonstrated, the MSC-based similarity index can distinguish the altered from the reference signals. Next, the variations between the reference cases of the three straight-edges are compared. MSC functions between the three straight-edge reference recordings were compared with each other, as shown on Figure V-16, before any holes or notches were cut into any of them. The plots of the MSC estimates do not show a clear frequency interval where the correlation between reference signals is strongest. Ideally, the MSC function would be close to a value of 1.0 from 20 to $80 \mathrm{kHz}$ for all three signal comparisons. The observable difference between the three reference cases is likely due to the coupling between transducers and specimens. Despite careful installation, there are still slight differences in coupling location and force. These differences are amplified in the recorded signal and distort the MSC coherence between identical pieces.

Nevertheless, there are individual spikes, or slices, with an MSC estimate close to 1.0, for all three comparisons. At around $69 \mathrm{kHz}$, the MSC values are high, consistently above 0.95 coherence. The slice appears to be insensitive to the coupling changes of the transducers. It exhibits the expected correlation between the reference straight-edge recordings, shown on Figure V-17. 

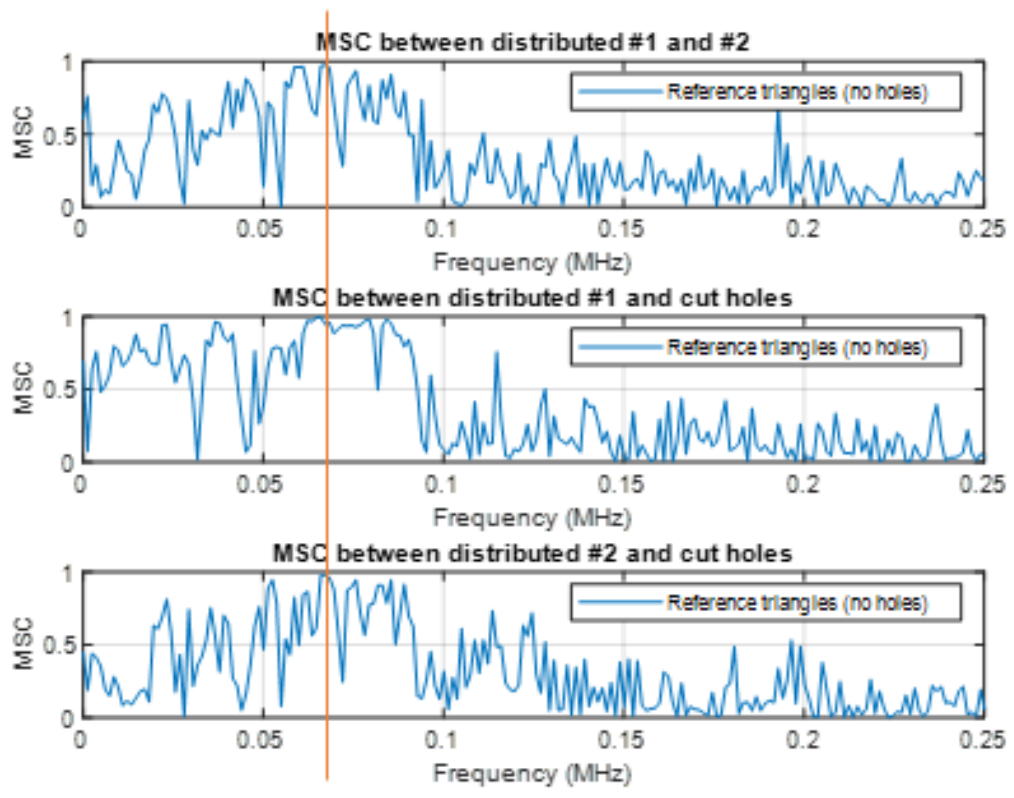

Figure V-16. Plots of MSC values between specimens (a) distributed holes \#1 vs distributed holes \#2, (b) distributed holes \#1 vs cut holes, and (c) distributed holes \#2 vs cut holes.

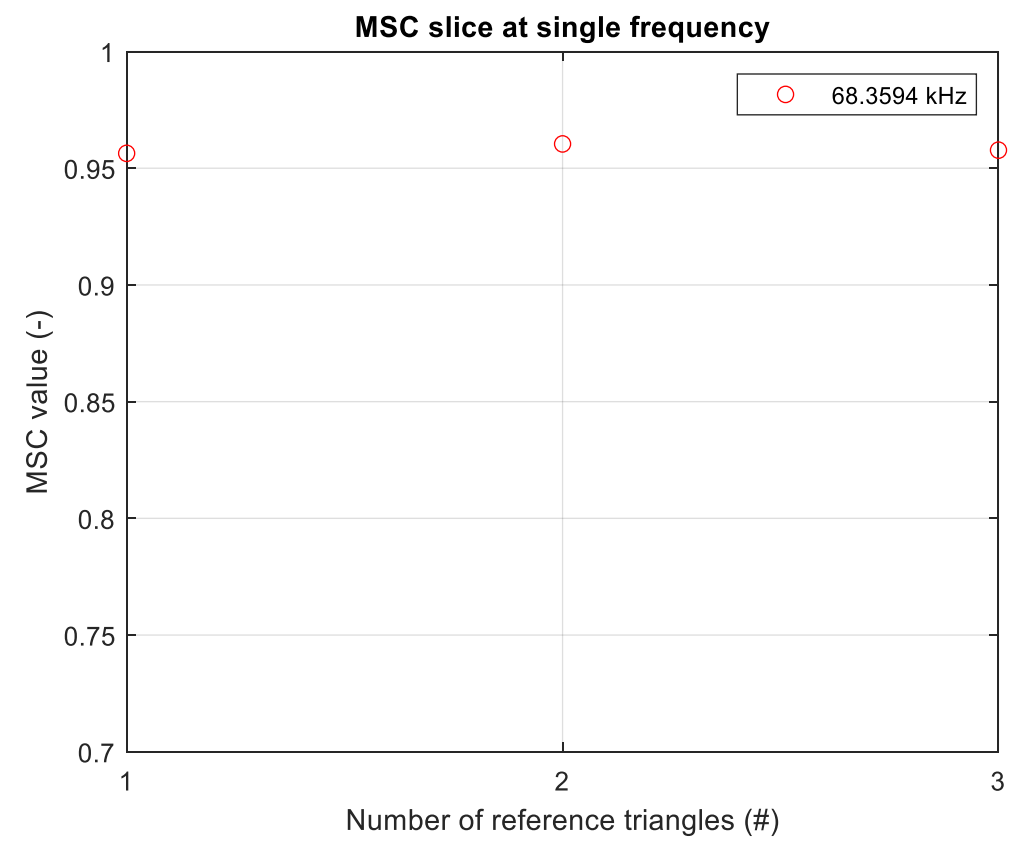

Figure V-17. Plot of MSC slice value for all three straight-edge reference recording comparisons.

The MSC slice similarity exists equally when comparing straight-edges experiencing identical alteration stages. The plastic pieces of Figure V-18 contain identical number and location of perforations at the five- and ten-holes stages, for the distributed holes (1) and (2) specimens, respectively. The MSC estimate between the two straight-edges at these stages yields a strong resemblance $(\mathrm{MSC}$ estimate $=0.97)$ at the $69 \mathrm{kHz}$ MSC slice. 

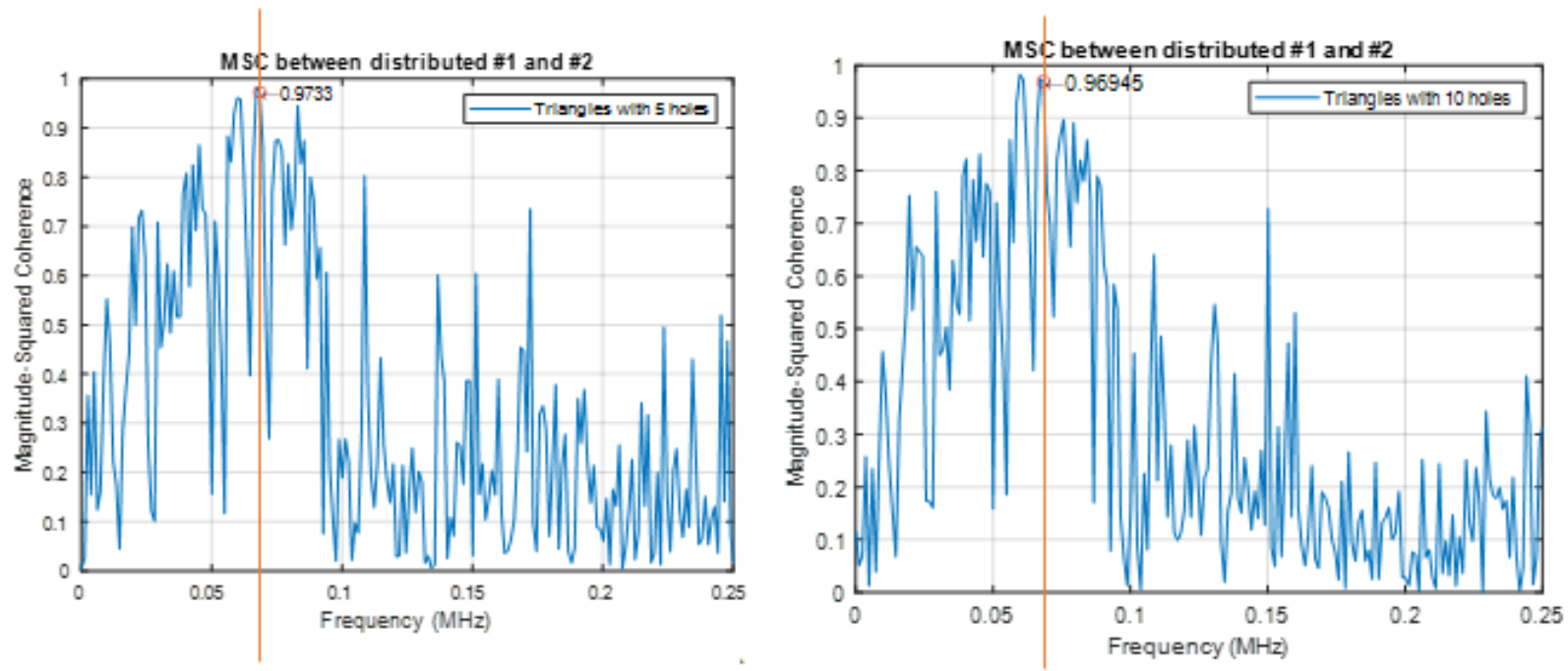

Figure V-18. Plots of MSC values between distributed holes \#1 and \#2 at the (a) five perforations and (b) ten perforations stages

However, this MCS slice is insensitive to alteration changes along with coupling changes. In Figure V-19, the MSC values are generated and plotted for three alteration cases of the distributed holes (1) specimen. The values are subsequently compared between each other using the MSC interval and MSC slice analysis. At $69 \mathrm{KHz}$, the MSC slice estimate remains consistently above 0.95 in Figure V-20 (b), despite the introduction of up to ten holes into the straight-edge triangle. The MSC slice does not register the introduction of holes in the distributed holes (1) specimen. In contrast, the MSC integral in Figure V-20 (a) across the 20 to $80 \mathrm{kHz}$ interval registers the decrease in MSC similarity, as mentioned before. 

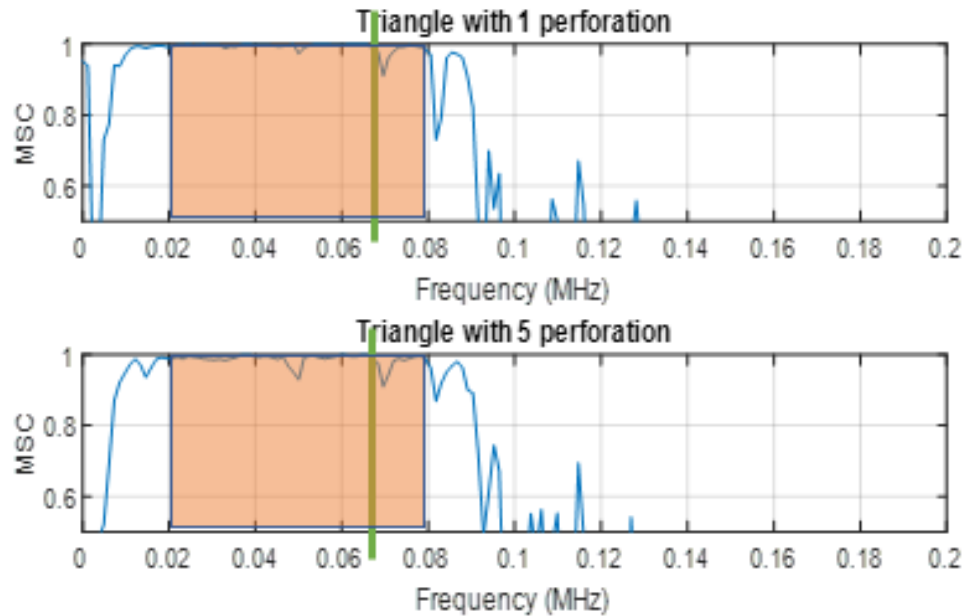

Triangle with 10 perforation

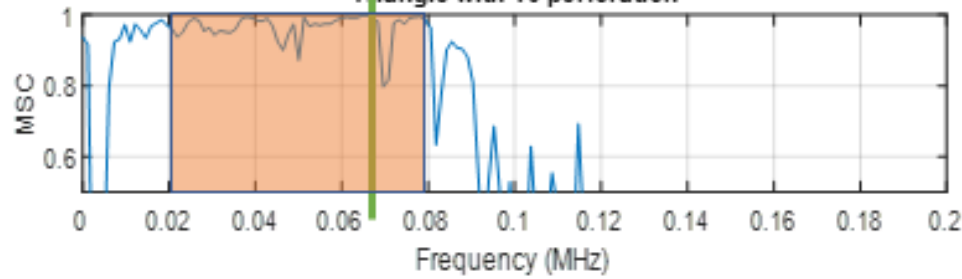

Figure V-19. Plots of MSC values for the distributed holes (1) sample at three different alteration stages. At each stage, MSC interval is highlighted (orange) and MSC slice (green) delineated. 
(a)

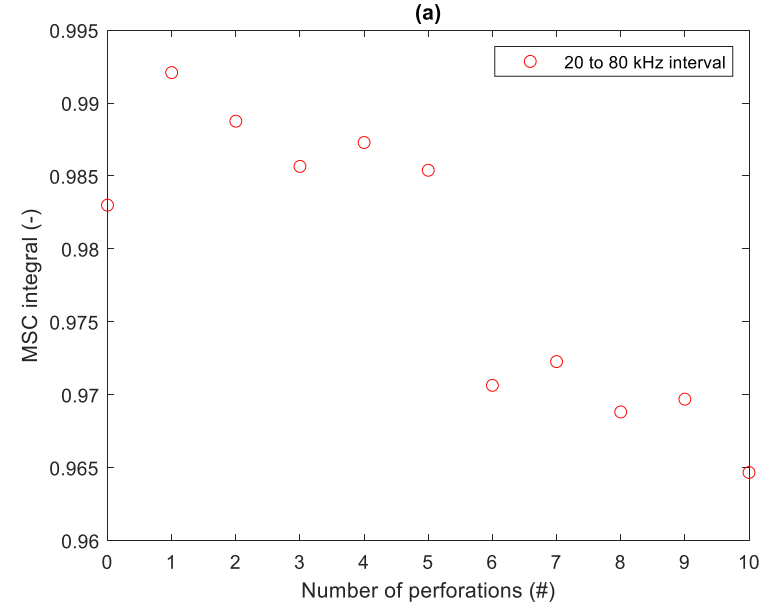

(b)

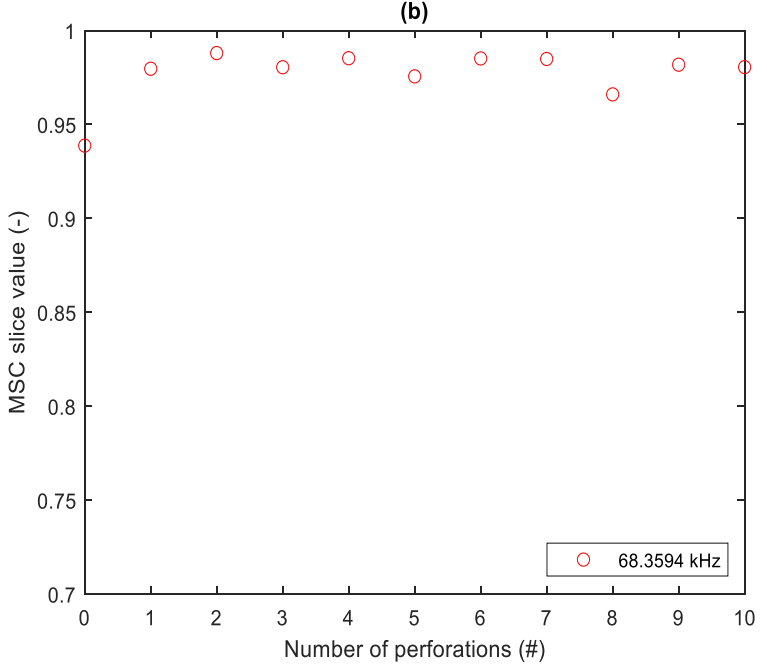

Figure V-20. Comparison of MSC slice (a) vs MSC integral (b) analysis across distributed holes \#1 straight-edge. 


\section{Summary and Conclusions}

This project aimed to evaluate the feasibility of an ultrasound-based tool to support quality control of manufactured parts using coda wave analysis. It picked proven capabilities of coda wave analysis for damage detection and applied it to commercially available mass manufactured parts. Two similarity indices, coefficient of determination, $R^{2}$ and an MSC-based index, were introduced and used to quantify the difference between a reference and an altered part. The introduced alterations were selected to be small compared to the pulse frequency and consisted of adding small magnets (for steel specimens) and drilling small holes (for plastic parts).

The study grants several valuable observations and lessons learned:

- Coda wave analysis is extremely sensitive in its ability to capture small differences between two parts. This is demonstrated through several experimental tests involving parts of different size, geometry, and materials. Most notably, alterations that are significantly smaller compared to the wavelength of the transmitted ultrasonic pulse can be detected.

- The most sensitive portion of a recorded ultrasonic signal is the coda wave portion, which follows the first arrival, or coherent portion of the signal. The reason for this that the stress wave has, at that point, interacted with many (or ideally: all) regions of a part, and thus contains the most information about it. This is in contrast to a traditional ultrasonic testing approach where only the first arrival (P-wave) is used, e.g. to determine the timeof-flight (TOF).

- The most suitable portion of a signal to be used for coda wave analysis is application dependent and has to be determined by visual inspection. Depending on how long data is recorded, it is not necessarily the last portion of the signal.

- The coda wave portion is able to capture small alterations for sampling rates that are even below the transmitted pulse frequency. This is promising as it allows for rapid and computationally-efficient analyses and inexpensive equipment to be employed.

- The MSC-based similarity indices are insensitive to small time shifts in the recorded signals and allows for selecting a frequency range of interest. The range should be selected upon visual inspection of the MSC function and can be over a range (computing the MSC integral) or one value (or slice) at a specific frequency.

- While both similarity indices were able to distinguish small differences between parts, the plastics straight-edge test demonstrates the remaining major challenge for the proposed approach to be successfully used as a quality control method: consistent transducer coupling across parts. While MSC might be able to compensate smaller variations in coupling, there are limitations.

Future work will include dealing with finding a solution to achieve consistent coupling between measurements. Alternatively, air-coupled ultrasonic systems could be evaluated. The challenge will still be to achieve consistent transducer locations with respect to the tested part. Also, air coupled systems have significantly lower SNR, which might be a challenge. Finally, machine learning might be useful in not only detecting alterations but characterizing them. 


\section{REFERENCES}

[1]. "In-Line Measurement and Control for Metals Processing." IOM3, 6 Dec. 2017, www.iom3.org/bulk-metal-forming-committee/event/inline-measurement-and-controlmetals-processing. Warwick University

[2]. Williams, Jacob, et al. "Defect Detection and Monitoring in Metal Additive Manufactured Parts through Deep Learning of Spatially Resolved Acoustic Spectroscopy Signals." Smart and Sustainable Manufacturing Systems, vol. 2, no. 1, Nov. 2018, p. 20180035. DOI.org (Crossref), doi:10.1520/SSMS20180035.

[3].Livings, Richard. “Quantitative Ultrasonic Coda Wave (Diffuse Field) NDE of Carbon-Fiber Reinforced Polymer Plates.” 2017, doi:10.31274/etd-180810-5180.

[4]. American Concrete Institute. Report on Nondestructive Test Methods for Evaluation of Concrete in Structures. American Concrete Institute, 2013.

[5]. Everton, Sarah K., et al. "Review of in-Situ Process Monitoring and in-Situ Metrology for Metal Additive Manufacturing." Materials \& Design, vol. 95, 2016, pp. 431-445., doi:10.1016/j.matdes.2016.01.099.

[6].Hafiz, Ali, and Thomas Schumacher. Monitoring of Applied Stress in Concrete Using Ultrasonic Full-Waveform Comparison Techniques. Edited by H. Felix Wu et al., 2017, p. 101692Z. DOI.org (Crossref), doi:10.1117/12.2267170.

[7].Zhang, Yuxiang, et al. "Three-Dimensional in-Situ Imaging of Cracks in Concrete Using Diffuse Ultrasound.” Structural Health Monitoring, vol. 17, no. 2, Mar. 2018, pp. 27984. DOI.org (Crossref), doi:10.1177/1475921717690938.

[8].Aki, Keiiti. "Analysis of the Seismic Coda of Local Earthquakes as Scattered Waves." Journal of Geophysical Research, vol. 74, no. 2, 1969, pp. 615-631., doi:10.1029/jb074i002p00615.

[9]. Chen, Andrew, and Thomas Schumacher. "Characterization of Flaws in Structural Steel Members Using Diffuse Wave Fields.” 2014, doi:10.1063/1.4864897.

[10]. Wang, Qiang, et al. "Damage Detection in Glass Fiber-Reinforced Plastics Using Ultrasonic Full-Waveform Comparison." Springer Proceedings in Physics Advances in Acoustic Emission Technology, 2019, pp. 155-163., doi:10.1007/978-3-030-12111-2_14.

[11]. Eric, Larose, and Hall Stephen. "Monitoring Stress Related Velocity Variation in Concrete with a $2 \times 10-5$ Relative Resolution Using Diffuse Ultrasound." The Journal of the Acoustical Society of America, vol. 125, no. 4, 2009, pp. 2641-2641., doi:10.1121/1.4784105. 
[12]. Zhang, Lei, et al. "Grease-like Coupling Agent Based on Metal Ionization Method for Low-Frequency Ultrasonic NDT." Results in Physics, vol. 12, Mar. 2019, pp. 1770-

76. DOI.org (Crossref), doi:10.1016/j.rinp.2019.01.084.

[13]. Ham, Suyun, and John S. Popovics. "Application of Contactless Ultrasound toward Automated Inspection of Concrete Structures." Automation in Construction, vol. 58, Oct. 2015, pp. 155-64. DOI.org (Crossref), doi:10.1016/j.autcon.2015.07.018.

[14]. Essig, W., et al. "Non-Contact Inline Monitoring of Thermoplastic CFRP Tape Quality Using Air-Coupled Ultrasound.” 2019, doi:10.1063/1.5084891.

[15]. Koester, L., et al. "Nondestructive Testing for Metal Parts Fabricated Using PowderBased Additive Manufacturing." Materials Evaluation, vol. 76, Apr. 2018.

[16]. Harley, Joel B., and Daniel Sparkman. Machine Learning and NDE: Past, Present, and Future. 2019, p. 090001. DOI.org (Crossref), doi:10.1063/1.5099819.

[17]. Rus, J., and C. U. Grosse. "Local Ultrasonic Resonance Spectroscopy: A Demonstration on Plate Inspection." Journal of Nondestructive Evaluation, vol. 39, no. 2, June 2020, p. 31. DOI.org (Crossref), doi:10.1007/s10921-020-00674-5.

[18]. Michaels, J. E., and T. E. Michaels. "Detection of Structural Damage from the Local Temporal Coherence of Diffuse Ultrasonic Signals." IEEE Transactions on Ultrasonics, Ferroelectrics and Frequency Control, vol. 52, no. 10, Oct. 2005, pp. 1769-82. DOI.org (Crossref), doi:10.1109/TUFFC.2005.1561631.

[19]. Kim, Do-Hyoung, et al. "Nondestructive Evaluation of Hidden Damages in Glass Fiber Reinforced Plastic by Using the Terahertz Spectroscopy.” International Journal of Precision Engineering and Manufacturing-Green Technology, vol. 4, no. 2, 2017, pp. 211-219., doi:10.1007/s40684-017-0026-x.

[20]. Manolakis, Dimitris G., et al. Statistical and Adaptive Signal Processing: Spectral Estimation, Signal Modeling, Adaptive Filtering, and Array Processing. McGraw-Hill, 2000.

[21]. Kolsky, H. "Stress Waves in Solids." Journal of Sound and Vibration, vol. 1, no. 1, Jan. 1964, pp. 88-110. DOI.org (Crossref), doi:10.1016/0022-460X(64)90008-2. 